LAPTH-1240/08

LPT-Orsay-08-29

\title{
Hexagon Wilson loop = six-gluon MHV amplitude
}

\author{
J.M. Drummond*, J. Henn*, G.P. Korchemsky** and E. Sokatchev* \\ * LAPTH1, Université de Savoie, CNRS; \\ 9 Chemin de Bellevue, B.P. 110, F-74941 Annecy-le-Vieux Cedex, France \\ ** Laboratoire de Physique Théoriqu£2, Université de Paris XI, \\ F-91405 Orsay Cedex, France
}

\begin{abstract}
We compare the two-loop corrections to the finite part of the light-like hexagon Wilson loop with the recent numerical results for the finite part of the MHV six-gluon amplitude in $\mathcal{N}=4$ SYM theory by Bern, Dixon, Kosower, Roiban, Spradlin, Vergu and Volovich (arXiv:0803.1465 [hep-th]) and demonstrate that they coincide within the error bars and, at the same time, they differ from the BDS ansatz by a non-trivial function of (dual) conformal kinematical invariants. This provides strong evidence that the Wilson loop/scattering amplitude duality holds in planar $\mathcal{N}=4$ SYM theory to all loops for an arbitrary number of external particles.
\end{abstract}

\footnotetext{
${ }^{1}$ Laboratoire de Physique Théorique d'Annecy-le-Vieux, UMR 5108

${ }^{2}$ Unité Mixte de Recherche du CNRS (UMR 8627)
} 


\section{Introduction}

In an important recent development in the study of the AdS/CFT correspondence, Alday and Maldacena proposed [1] the strong coupling description of planar gluon scattering amplitudes in the maximally supersymmetric $\mathcal{N}=4$ Yang-Mills (SYM) theory and were able to make a direct comparison with a prediction based on weak coupling results for the same amplitudes. At weak coupling, following years of intensive studies of gluon scattering amplitudes [2, 3, 4], the conjecture was put forward by Bern, Dixon and Smirnov [5] (see also an earlier version in [6]) that the maximally helicity-violating (MHV) planar gluon amplitudes in $\mathcal{N}=4$ SYM have a remarkably simple all-loop iterative structure. In general, these amplitudes have the following form:

$$
\ln \mathcal{M}_{n}^{(\mathrm{MHV})}=[\mathrm{IR} \text { divergences }]+F_{n}^{(\mathrm{MHV})}\left(p_{1}, \ldots, p_{n} ; a\right)+O(\epsilon) .
$$

Here $\mathcal{M}_{n}^{(\mathrm{MHV})}$ is the color-ordered planar gluon amplitude, divided by the tree amplitude. The first term on the right-hand side describes the infrared (IR) divergences and the second term is the finite contribution dependent on the gluon momenta $p_{i}$ and on the 't Hooft coupling $a=g^{2} N /\left(8 \pi^{2}\right)$. The structure of IR divergences is well understood in any gauge theory [7]. In particular, in theories with a vanishing beta function like $\mathcal{N}=4 \mathrm{SYM}$, the leading IR singularity in dimensional regularization is a double pole, whose coefficient is the universal cusp anomalous dimension appearing in many physical processes [8, 9, 10, 11]. The BDS conjecture provides an explicit expression for the finite part, $F_{n}^{(\mathrm{MHV})}=F_{n}^{(\mathrm{BDS})}$, for an arbitrary number $n$ of external gluons, to all orders in the coupling $a$. Remarkably, the dependence of $F_{n}^{(B D S)}$ on the kinematical invariants is described by a function which is coupling independent and, therefore, can be determined at one loop. At present, the BDS conjecture has been tested up to three loops for $n=4$ [5] and up to two loops for $n=5$ [12. The explicit investigation of the conjecture for $n=6$ at two loops is the subject of this paper and of the parallel paper [13].

As mentioned earlier, after the work of Alday and Maldacena it became possible to test the BDS conjecture at strong coupling. According to their proposal, at strong coupling the planar gluon amplitude is related to the area of a minimal surface in $\mathrm{AdS}_{5}$ space attached to a specific closed contour $C_{n}$, made out of $n$ light-like segments $\left[x_{i}, x_{i+1}\right]$ defined by the gluon momenta $x_{i}^{\mu}-x_{i+1}^{\mu}=p_{i}^{\mu}$ (with the cyclicly condition $x_{n+1} \equiv x_{1}$ ),

$$
\ln \mathcal{M}_{n}=-\frac{\sqrt{g^{2} N}}{2 \pi} A_{\min }\left(C_{n}\right) \text {. }
$$

For $n=4$ the minimal surface $A_{\min }\left(C_{4}\right)$ was found explicitly in [1], by making use of the conformal symmetry of the problem. With the appropriate AdS equivalent of dimensional regularization, the divergent part of $\ln \mathcal{M}_{4}$ has the expected pole structure, with the coefficient in front of the double pole given by the known strong coupling value of the cusp anomalous dimension. Most importantly, the finite part of $\ln \mathcal{M}_{4}$ is in perfect agreement with $F_{4}^{(\mathrm{BDS})}$ from the BDS ansatz. For $n \geq 5$ the practical evaluation of the solution of the classical string equations turns out to be difficult, but it simplifies significantly for $n$ large [14]. In the limit $n \rightarrow \infty$ the strong coupling prediction for $\ln \mathcal{M}_{n}$ disagrees with the BDS ansatz. This indicates [14] that the BDS conjecture should fail for a sufficiently large number of gluons and/or at sufficiently high loop level.

Alday and Maldacena pointed out [1] that their prescription (2) is mathematically equivalent to the strong coupling calculation of the expectation value of a Wilson loop $W\left(C_{n}\right)$, defined on the light-like contour $C_{n}$ [15, 16]. This should not come as a total surprise, since the intimate 
relationship between the infrared divergences of the scattering of massless particles and the ultraviolet divergences of Wilson loops with cusps is well known in QCD [8, 9, 10]. Inspired by this, in [17] three of us conjectured that a similar duality relation between planar gluon amplitudes and light-like Wilson loops also exists at weak coupling. We illustrated this duality by an explicit one-loop calculation in the simplest case $n=4$. This was later extended to the case of arbitrary $n$ at one loop in [18. If we write the log of the Wilson loop in the following way,

$$
\ln W(C)=[\mathrm{UV} \text { divergences }]+F_{n}^{(\mathrm{WL})}\left(x_{1}, \ldots, x_{n} ; a\right)+O(\epsilon),
$$

then the duality relation identifies the finite parts of the two objects up to an additive constant, once one imposes the relations $p_{i}^{\mu}=x_{i}^{\mu}-x_{i+1}^{\mu}$,

$$
F_{n}^{(\mathrm{MHV})}=F_{n}^{(\mathrm{WL})}+\text { const } .
$$

This property is extremely non-trivial.

Further evidence in favor of the duality relation (44) at weak coupling came from two-loop calculations of $\ln W\left(C_{n}\right)$ for $n=4$ [20] and $n=5$ [21]. Our results were in agreement with the two-loop MHV gluon amplitude calculations [6, 12, and hence with the BDS ansatz for $n=4,5$. Furthermore, in [20] we proposed and in [21] we proved a conformal Ward identity for the light-like Wilson loop $W\left(C_{n}\right)$, valid to all orders in the coupling. It fixes the functional form of the finite part of $\ln W\left(C_{n}\right)$ for $n=4$ and $n=5$, up to an additive constant, to agree with the conjectured BDS form for the corresponding gluon amplitudes 1 However, for $n \geq 6$, although the BDS ansatz $F_{n}^{(\mathrm{BDS})}$ satisfies the conformal Ward identity, $F_{n}^{(\mathrm{WL})}$ is allowed to differ from $F_{n}^{(\mathrm{BDS})}$ by an arbitrary function of conformal invariants (for $n=6$ there are three such invariants). This result provided a possible explanation of the BDS conjecture for $n=4,5$ (assuming that the MHV amplitudes have the same conformal properties as the Wilson loop, see the discussion in Sect. 2.4 below), but left the door open for potential deviations from it for $n \geq 6$. To verify whether the BDS conjecture and/or the proposed duality relation (4) still hold for $n=6$ to two loops, it was necessary to perform explicit two-loop calculations of the finite parts of the six-gluon amplitude $F_{6}^{(\mathrm{MHV})}$, and of the hexagon Wilson loop $F_{6}^{(\mathrm{WL})}$.

In a recent paper [24] we reported on the two-loop calculation of $F_{6}^{(\mathrm{WL})}$ and found that it differs from the BDS ansatz,

$$
F_{6}^{(\mathrm{WL})}=F_{6}^{(\mathrm{BDS})}+R_{6}
$$

Here, in complete agreement with the conformal Ward identity, $R_{6}$ is a non-trivial 'remainder' function of three conformally invariant combinations of the kinematical variables. As was emphasized in [24], were the duality relation (44) to hold for $n=6$, the function $R_{6}$ would describe the discrepancy between the BDS ansatz and the scattering amplitude. A parallel two-loop six-gluon amplitude calculation has been undertaken by Bern, Dixon, Kosower, Roiban, Spradlin, Vergu and Volovich and the results became available very recently [13].

The detailed numerical comparisons of the two calculations, described in this paper and in the parallel publication [13], shows that, firstly, the BDS ansatz fails for $n=6$ at two loops and, secondly, the duality with Wilson loops is preserved,

$$
F_{6}^{(\mathrm{MHV})}=F_{6}^{(\mathrm{WL})}-c_{6}(a),
$$

\footnotetext{
${ }^{1}$ Later, similar Ward identities were also obtained at strong coupling using the AdS/CFT correspondence in Refs. [22, 23].
} 
where $c_{6}(a)$ is a constant. We consider this as very strong evidence that the duality relation (4) should hold for arbitrary $n$ to all orders in the coupling.

The real challenge now is, in our opinion, to find out the deep reason behind the surprising duality (44) between two apparently unrelated objects in the $\mathcal{N}=4$ theory. One might speculate that the scattering amplitudes and the light-like Wilson loops share the same (probably infinite) set of symmetries, of which (dual) conformal symmetry is just the most visible part. If so, these symmetries may completely fix the form of the quantities on both sides of the duality relation. This could be the manifestation of some new type of integrability of $\mathcal{N}=4$ SYM theory. We would like to stress that the duality relation (4) only holds in the planar limit. Indeed, the known two-loop non-planar contributions to the four-gluon amplitude [3] appear to break dual conformal symmetry, which, as we have shown, is an important ingredient of the duality. Also, recent studies in the AdS/CFT correspondence suggest [25, 26, 27] that at strong coupling the duality relation (4) can be extended to scattering amplitudes involving gluinos (both in the $\mathcal{N}=4 \mathrm{SYM}$ and its deformed versions), as well as to the on-shell matrix elements of conserved currents [14]. It would be interesting to verify whether the same relation holds at weak coupling. This question is beyond the scope of the present paper and deserves further investigation.

We would like to point out that a weaker form of the duality (4) has already been observed in QCD in the special, high-energy (Regge) limit $s \gg-t>0$ for the four-gluon amplitude up to two loops [10]. The same relationship holds in any gauge theory ranging from QCD to $\mathcal{N}=4$ SYM. The essential difference between these theories is that in the former case the duality is only valid in the Regge limit, whereas in the latter case it is exact in general kinematics. Moreover, in Ref. [17, based on the BDS ansatz, three of us argued that the four-gluon amplitude is Regge exact in $\mathcal{N}=4 \mathrm{SYM}$. Namely, the contribution of the gluon Regge trajectory to $\ln \mathcal{M}_{4}$ coincides with its exact expression evaluated for arbitrary values of $s$ and $t$, up to terms vanishing as $\epsilon \rightarrow 0$. This property allowed us to obtain the explicit expression for the three-loop gluon Regge trajectory in $\mathcal{N}=4 \mathrm{SYM}$ [17]. The two-loop correction to this trajectory was found to be in agreement with the results of Ref. [28] and the three-loop correction was later confirmed in Refs. [29, 30, 31, 32]. We would like to mention that a thorough analysis of the Regge limit of planar multi-gluon amplitudes in $\mathcal{N}=4$ SYM was recently performed in Refs. [30, 31] and it provided further evidence that the BDS ansatz needs to be corrected [31].

The paper is organized as follows. In Section 2 we explain in detail the proposed duality between planar gluon amplitudes and light-like Wilson loops. We summarize some basic facts about planar gluon amplitudes, focusing on the structure of their infrared divergences and on the BDS ansatz for the finite part. Next we describe the light-like Wilson loop, the structure of its ultraviolet singularities and the anomalous conformal Ward identity for its finite part. Then we state the duality relation and discuss its consequences for the six-gluon amplitude. Section 3 contains a description of our two-loop calculation of the hexagon Wilson loop $W\left(C_{6}\right)$ (already reported in [24]). We separate the divergent and finite part of individual Feynman diagrams by employing the 'subtraction procedure' proposed in [21, and then demonstrate that the divergent part of $\ln W\left(C_{6}\right)$ is of the expected form. We work out the representation for the finite part $F_{6}^{(\mathrm{WL})}$ in the form of convergent multiple parameter integrals which can easily be evaluated numerically for given kinematical configurations. In Section 4 we perform a detailed numerical comparison of our results of the hexagon Wilson loop calculation with the BDS ansatz for $n=6$ and with the results of the parallel six-gluon calculation [13]. Section 5 contains concluding remarks. 

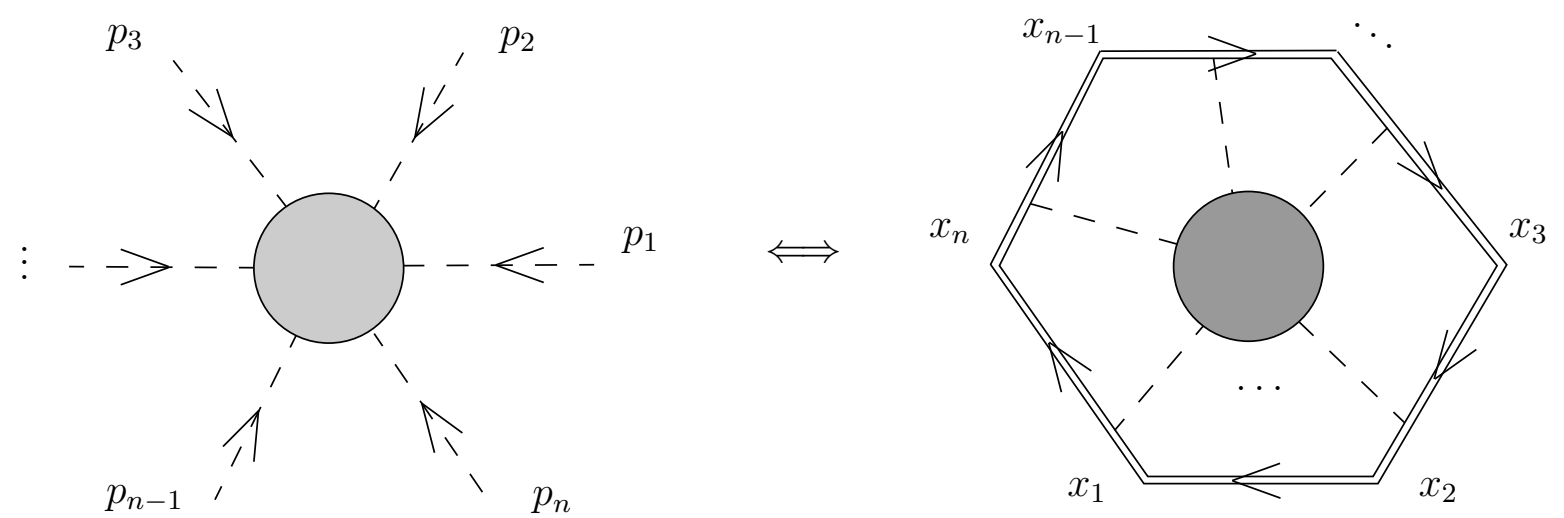

Figure 1: The conjectured duality relation between the gluon scattering amplitude $\mathcal{M}_{n}$ and the Wilson loop $W\left(C_{n}\right)$. The dashed lines depict gluons and the double lines the integration contour $C_{6}$. The momenta of the incoming gluons are identified as the light-like segments of the integration contour, $p_{i} \sim x_{i}-x_{i+1}$.

\section{Planar gluon amplitude/Wilson loop duality}

Recent studies revealed that the gluon scattering amplitudes have a number of remarkable properties in the $\mathcal{N}=4 \mathrm{SYM}$ theory. To describe them, we first recall some general features of such amplitudes.

\subsection{Planar amplitudes}

In a generic Yang-Mills theory with an $S U(N)$ gauge group, the scattering amplitude of $n$ gluons can be decomposed into color-ordered partial amplitudes multiplied by the corresponding color structure [33]. In the planar limit, the dominant contribution only comes from the single-trace color structures leading to

$$
\mathcal{A}_{n}\left(\left\{p_{i}, h_{i}, a_{i}\right\}\right)=2^{n / 2} g^{n-2} \sum_{\sigma \in S_{n} / Z_{n}} \operatorname{tr}\left[t^{a_{\sigma(1)}} \ldots t^{a_{\sigma(n)}}\right] A_{n}\left(\sigma\left(1^{h_{1}}, \ldots, n^{h_{n}}\right)\right)+O\left(1 / N^{2}\right)
$$

where each gluon is characterized by its on-shell momentum $p_{i}^{\mu}\left(p_{i}^{2}=0\right)$, helicity $h_{i}= \pm 1$ and color index $a_{i}$. Here the sum runs over all possible non-cyclic permutations $\sigma$ of the set $\{1, \ldots, n\}$ and the color trace involves the generators $t^{a}$ of $S U(N)$ in the fundamental representation normalized as $\operatorname{tr}\left(t^{a} t^{b}\right)=\frac{1}{2} \delta^{a b}$. All gluons are treated as incoming, so that the momentum conservation takes the form $\sum_{i=1}^{n^{2}} p_{i}=0$.

The planar scattering amplitude (7) is uniquely determined by the set of color-ordered amplitudes $A_{n}$. It follows from the supersymmetric Ward identities [34] that the amplitude $A_{n}$ vanishes to all orders when either all the external gluons or all gluons but one have the same helicity. When two gluons have, e.g., negative helicity and the remaining $n-2$ gluons have positive helicity, the so-called maximally helicity-violating (MHV) amplitudes have the following remarkably simple form in the spinor helicity formalism:

$$
A_{n}\left(1^{+} \ldots m_{1}^{-} \ldots m_{2}^{-} \ldots n^{+}\right)=i \frac{\left\langle m_{1} m_{2}\right\rangle^{4}}{\langle 12\rangle\langle 23\rangle \ldots\langle n 1\rangle} \mathcal{M}_{n}^{(\mathrm{MHV})}
$$


Here the helicity structure is described by massless Weyl spinors $u_{ \pm}(p)$ of momentum $p$ and chirality \pm 1 ; they form Lorentz invariant 'inner products' of the type $\langle j k\rangle=\left\langle j^{-} \mid k^{+}\right\rangle=\bar{u}_{-}\left(p_{j}\right) u_{+}\left(p_{k}\right)$. The Lorentz scalar $\mathcal{M}_{n}=1+a \mathcal{M}_{n ; 1}+O\left(a^{2}\right)$ does not depend on the positions $m_{1,2}$ of the negative-helicity gluons [35]. According to (7) and (8), the perturbative corrections to the planar MHV amplitude are determined to all loops by the single function $\mathcal{M}_{n}^{(\mathrm{MHV})}$ of the Mandelstam variables. This function (more precisely, its logarithm) is the main object of interest in this paper.

It is worthwhile mentioning that for scattering amplitudes with more than two negative helicities (next-to-MHV amplitudes and their generalizations) the situation is more complex. At tree level, in the spinor helicity formalism these amplitudes are expressed in terms of various Lorentz structures built from spinors [33]. At one-loop level, however, the perturbative corrections to the non-MHV amplitudes induce new Lorentz structures 4. As a result, in distinction with (8), the non-MHV amplitudes do not admit a simple factorized form. This is the reason why the duality (4) with Wilson loops that we discuss here only concerns MHV amplitudes.

\subsection{Planar MHV amplitudes}

The gluon scattering amplitudes suffer from infrared divergences. In supersymmetric gauge theories, they can be regularized using the dimensional reduction scheme (DRED) with $D=$ $4-2 \epsilon_{\mathrm{IR}}$ and $\epsilon_{\mathrm{IR}}<0$. 2 In this case, the IR divergences appear as poles in $\epsilon_{\mathrm{IR}}$. At a given loop order $l$, the maximal order of the poles is $2 l$. In a generic Yang-Mills theory, the IR divergent part of the planar scattering amplitudes has a universal form which is sensitive neither to the helicities of the scattered particles, nor to their type (gluons, fermions, scalars). More precisely, the IR divergences exponentiate in the all-loop planar amplitude and, as a consequence, they can be factorized into a universal divergent factor. In application to the MHV planar amplitudes in $\mathcal{N}=4$ SYM theory, this property allows one to decompose $\ln \mathcal{M}_{n}^{(\mathrm{MHV})}$ into divergent and finite parts as follows,

$$
\ln \mathcal{M}_{n}^{(\mathrm{MHV})}=Z_{n}+F_{n}^{(\mathrm{MHV})}+O\left(\epsilon_{\mathrm{IR}}\right),
$$

In a theory with a vanishing beta function, the IR divergent part $Z_{n}$ takes the particularly simple form

$$
Z_{n}=-\frac{1}{4} \sum_{l \geq 1} a^{l}\left(\frac{\Gamma_{\text {cusp }}^{(l)}}{\left(l \epsilon_{\mathrm{IR}}\right)^{2}}+\frac{G^{(l)}}{l \epsilon_{\mathrm{IR}}}\right) \sum_{i=1}^{n}\left(-\frac{t_{i}^{[2]}}{\mu_{\mathrm{IR}}^{2}}\right)^{-l \epsilon_{\mathrm{IR}}}
$$

where $t_{i}^{[2]} \equiv s_{i, i+1}=\left(p_{i}+p_{i+1}\right)^{2}$ is the invariant mass of two adjacent gluons with indices $i$ and $i+1$ subject to the periodicity condition $i+n \equiv i$. The IR cut-off $\mu_{\mathrm{IR}}^{2}$ is related to the dimensional regularization scale $\mu^{2}$ as

$$
\mu_{\mathrm{IR}}^{2}=4 \pi \mathrm{e}^{-\gamma_{E}} \mu^{2}
$$

where $\gamma_{E}$ is the Euler constant.

We did not put the superscript MHV on $Z_{n}$ in order to indicate that it has a universal form for all planar amplitudes in the $\mathcal{N}=4$ SYM theory. The coefficients of the IR poles in (10) are the expansion coefficients of the cusp anomalous dimension $\Gamma_{\text {cusp }}(a)=\sum_{l=1}^{\infty} a^{l} \Gamma_{\text {cusp }}^{(l)}$ and of the

\footnotetext{
${ }^{2}$ More precisely, a modification of the DRED scheme, the so-called four-dimensional helicity (FDH) scheme, has been used in [36.
} 
so-called collinear anomalous dimension $G(a)=\sum_{l=1}^{\infty} a^{l} G^{(l)}$. To two-loop order, they are given in the DRED scheme by

$$
\Gamma_{\text {cusp }}(a)=2 a-2 \zeta_{2} a^{2}+O\left(a^{3}\right), \quad G(a)=-\zeta_{3} a^{2}+O\left(a^{3}\right) .
$$

By definition [37, 8, 38], the cusp anomalous dimension $\Gamma_{\text {cusp }}(a)$ describes specific ultraviolet divergences of a Wilson loop evaluated over a contour with a cusp. Its appearance in the infrared divergent part of the scattering amplitude (10) is not accidental, of course. It has its roots in the deep relation between scattering amplitudes in gauge theory and Wilson loops evaluated over specific contours in Minkowski space-time, defined by the particle momenta [8, 9, 10]. It should be mentioned that this relation is not specific to $\mathcal{N}=4 \mathrm{SYM}$ and it holds in any gauge theory, including QCD. The two-loop expression for $\Gamma_{\text {cusp }}(a)$ in a generic (supersymmetric) Yang-Mills theory was found in Refs. [38, 11]. We remark that in $\mathcal{N}=4 \mathrm{SYM}$ theory, $\Gamma_{\text {cusp }}(a)$ is known at weak coupling to four loops [39], and there is a conjecture for it to all loops [40]. At strong coupling, the solution to the BES equation proposed in [40] produces a strong coupling expansion of $\Gamma_{\text {cusp }}(a)$ [41, 42, 43]. The first few terms of this expansion are in agreement with the existing quantum superstring calculation of Refs. [44, 45, 46]. The non-universal collinear anomalous dimension $G(a)$ is known to four loops at weak coupling [5, 47].

Let us now examine the finite part of the MHV planar amplitude $F_{n}^{(\mathrm{MHV})}$. In a generic YangMills theory, it has a much more complicated form compared to the divergent part. Surprisingly, this finite part becomes considerably simpler in $\mathcal{N}=4$ SYM. Previously, the finite parts of the following MHV planar amplitudes have been calculated:

- $n=4$ gluon amplitude up to three loops [3, 6, 5];

- $n=5$ gluon amplitude up to two loops [12];

- $n \geq 6$ gluon amplitude at one loop [2].

These calculations revealed a remarkable iterative structure of $F_{n}^{(\mathrm{MHV})}$. They led to the formulation of the Bern-Dixon-Smirnov ansatz (BDS) which provides a conjectured expression for the finite part of the MHV planar amplitudes valid for an arbitrary number of gluons $n \geq 4$ and to all orders in the coupling. The BDS ansatz reads

$$
F_{n}^{(\mathrm{BDS})}=\frac{1}{2} \Gamma_{\text {cusp }}(a) \mathcal{F}_{n}+c(a)
$$

where $\Gamma_{\text {cusp }}(a)$ is the cusp anomalous dimension defined in (12) and $c(a)=-\frac{1}{2} \zeta_{2}^{2} a^{2}+O\left(a^{3}\right)$ is a universal (independent of $n$ ) additive constant. The dependence on the kinematical invariants is described by the function $\mathcal{F}_{n}$. According to the BDS conjecture, this function is coupling independent, and can thus be determined at one loop. For our purposes here we only need its explicit expressions for $n=4,5,6$ :

$$
\begin{aligned}
& \mathcal{F}_{4}=\frac{1}{2} \ln ^{2}\left(\frac{t_{1}^{[2]}}{t_{2}^{[2]}}\right)+4 \zeta_{2}, \\
& \mathcal{F}_{5}=\frac{1}{2} \sum_{i=1}^{5}\left[-\frac{1}{2} \ln \left(\frac{t_{i}^{[2]}}{t_{i+3}^{[2]}}\right) \ln \left(\frac{t_{i+1}^{[2]}}{t_{i+2}^{[2]}}\right)+\frac{3}{2} \zeta_{2}\right], \\
& \mathcal{F}_{6}=\frac{1}{2} \sum_{i=1}^{6}\left[-\ln \left(\frac{t_{i}^{[2]}}{t_{i}^{[3]}}\right) \ln \left(\frac{t_{i+1}^{[2]}}{t_{i}^{[3]}}\right)+\frac{1}{4} \ln ^{2}\left(\frac{t_{i}^{[3]}}{t_{i+1}^{[3]}}\right)-\frac{1}{2} \operatorname{Li}_{2}\left(1-\frac{t_{i}^{[2]} t_{i+3}^{[2]}}{t_{i}^{[3]} t_{i+2}^{[3]}}\right)+\frac{3}{2} \zeta_{2}\right],
\end{aligned}
$$


where $t_{i}^{[r]}=\left(p_{i}+\ldots+p_{i+r-1}\right)^{2}$ are the Mandelstam kinematical invariants.

\section{$2.3 \quad$ Light-like Wilson loops}

Let us now turn to the description of the light-like Wilson loops, which are the counterparts of the MHV planar amplitudes in the duality relation discussed in this paper. In the $\mathcal{N}=4 \mathrm{SYM}$ theory with an $S U(N)$ gauge group they are defined as

$$
W\left(C_{n}\right)=\frac{1}{N}\left\langle 0\left|\operatorname{Tr} \mathrm{P} \exp \left(i \oint_{C_{n}} d x^{\mu} A_{\mu}(x)\right)\right| 0\right\rangle,
$$

where the gauge field $A_{\mu}(x)$ is integrated along the contour $C_{n}=\bigcup_{i=1}^{n} \ell_{i}$ made out of $n$ light-like segments joining the cusp points $x_{i}^{\mu}$ (with $\left.i=1,2, \ldots, n\right)$

$$
\ell_{i}=\left\{x^{\mu}\left(\tau_{i}\right)=\tau_{i} x_{i}^{\mu}+\left(1-\tau_{i}\right) x_{i+1}^{\mu} \mid \tau_{i} \in[0,1]\right\}
$$

such that the tangent vectors $\partial_{\tau_{i}} x^{\mu}\left(\tau_{i}\right)=x_{i, i+1}^{\mu}$ are light-like, $x_{i, i+1}^{2}=0$. The symbol $\mathrm{P}$ indicates the ordering of the $S U(N)$ indices along the integration contour $C_{n}$.

The Wilson loop (17) is a gauge invariant quantity depending on the integration contour $C_{n}$. As a function of the cusp points, it is invariant under their cyclic permutations and flips,

$$
W\left(x_{1}, x_{2}, \ldots, x_{n}\right)=W\left(x_{n}, x_{1}, \ldots, x_{n-1}\right)=W\left(x_{n}, x_{n-1}, \ldots, x_{1}\right) .
$$

If the contour $C_{n}$ did not have cusps, the Wilson loop would be a finite quantity in $D=$ 4 [37, 48, 19]. Characteristic feature of the light-like contour $C_{n}$ is that it maps to a similar contour under $S O(2,4)$ conformal transformations. As a result, a finite Wilson loop would be a conformal invariant function of $x_{i}[1,20]$. We would like to stress that, contrary to the gluon scattering amplitudes, the Wilson loop (17) is defined in configuration space and the conformal transformations act on the four-vectors $x_{i}^{\mu}$ defining the positions of the cusp points in Minkowski space-time. Due to the presence of cusps on the integration contour $C_{n}$, the Wilson loop (17) has specific ultraviolet divergences [37, 48, 8, 38, 19, 9] which make the conformal symmetry of $W\left(C_{n}\right)$ anomalous.

To regularize the cusp singularities, we use dimensional reduction with $D=4-2 \epsilon_{\mathrm{Uv}}$ and $\epsilon_{\mathrm{UV}}>0$ (notice the sign difference compared to $\epsilon_{\mathrm{IR}}$ ). Like the scattering amplitude, the Wilson loop can be split into a divergent and a finite part,

$$
\ln W\left(C_{n}\right)=Z_{n}^{(\mathrm{WL})}+F_{n}^{(\mathrm{WL})}+O\left(\epsilon_{\mathrm{UV}}\right) .
$$

The divergent part $Z_{n}^{(\mathrm{WL})}$ has the special form [19]

$$
Z_{n}^{(\mathrm{WL})}=-\frac{1}{4} \sum_{l \geq 1} a^{l}\left(\frac{\Gamma_{\mathrm{cusp}}^{(l)}}{\left(l \epsilon_{\mathrm{UV}}\right)^{2}}+\frac{\Gamma^{(l)}}{l \epsilon_{\mathrm{UV}}}\right) \sum_{i=1}^{n}\left(-x_{i, i+2}^{2} \mu_{\mathrm{UV}}^{2}\right)^{l \epsilon_{\mathrm{UV}}}
$$

where $\Gamma_{\text {cusp }}^{(l)}$ are the expansion coefficients of the cusp anomalous dimension (12) and $\Gamma^{(l)}$ is the counterpart of the collinear anomalous dimension entering in (10),

$$
\Gamma(a)=\sum_{l \geq 1} a^{l} \Gamma^{(l)}=-7 \zeta_{3} a^{2}+O\left(a^{3}\right) .
$$


The UV cut-off $\mu_{\mathrm{UV}}^{2}$ is related to the dimensional regularization scale $\mu^{2}$ as (cf. (11))

$$
\mu_{\mathrm{UV}}^{2}=\pi \mathrm{e}^{\gamma_{E}} \mu^{2}
$$

The finite part of the Wilson loop, $F_{n}^{(\mathrm{WL})}$, does not depend on the renormalization scale $\mu_{\mathrm{UV}}^{2}$ and it is a dimensionless function of the distances $x_{i j}^{2}$ with $i, j=1, \ldots, n$. Since the edges of $C_{n}$ are light-like, $x_{i, i+1}^{2}=0$, the non-vanishing distances are $x_{i j}^{2}$ with $|i-j| \geq 2$. Despite the fact that the conformal invariance of $W\left(C_{n}\right)$ is broken by the cusp singularities, it imposes severe constraints on $F_{n}^{(\mathrm{WL})}$. We showed in [20, 21] that $F_{n}^{(\mathrm{WL})}$ has to satisfy the following anomalous conformal Ward identity:

$$
K^{\mu} F_{n}^{(\mathrm{WL})} \equiv \sum_{i=1}^{n}\left(2 x_{i}^{\nu} x_{i} \cdot \partial_{i}-x_{i}^{2} \partial_{i}^{\nu}\right) F_{n}^{(\mathrm{WL})}=\frac{1}{2} \Gamma_{\text {cusp }}(a) \sum_{i=1}^{n} x_{i, i+1}^{\nu} \ln \left(\frac{x_{i, i+2}^{2}}{x_{i-1, i+1}^{2}}\right) .
$$

The differential operator on the left-hand side is the conformal boost (special conformal transformation) generator $K^{\mu}$. The right-hand side expresses the conformal anomaly due to the cusp singularities. As explained in [21], it has a universal functional form, with the coupling dependence coming only through the cusp anomalous dimension $\Gamma_{\text {cusp }}(a)$. For $n=4$ and $n=5$ this relation is powerful enough to determine the all-loop expressions for $F_{4}^{(\mathrm{WL})}$ and $F_{5}^{(\mathrm{WL})}$ up to an additive coupling-dependent constant. The reason for this is that any potential solution of the homogeneous differential equation $K^{\mu} F_{n}^{(\mathrm{WL})}=0$ would be a conformal invariant. It is well known that such invariants take the form of cross-ratios $x_{i j}^{2} x_{k l}^{2} /\left(x_{i k}^{2} x_{j l}^{2}\right)$. It is then immediately clear that one cannot build invariants from four or five points $x_{i}^{\mu}$ with light-like separations $x_{i, i+1}^{2}=0$. However, they can be constructed starting from six points. In particular, for the hexagon Wilson loop $W\left(C_{6}\right)$ there are three such cross-ratios,

$$
u_{1}=\frac{x_{13}^{2} x_{46}^{2}}{x_{14}^{2} x_{36}^{2}}, \quad u_{2}=\frac{x_{24}^{2} x_{15}^{2}}{x_{25}^{2} x_{14}^{2}}, \quad u_{3}=\frac{x_{35}^{2} x_{26}^{2}}{x_{36}^{2} x_{25}^{2}} .
$$

As a result, for $n \geq 6$ the general solution of the conformal Ward identity (24) will contain an arbitrary function of the conformal cross-ratios. This function was calculated at two loops for $n=6$ in [24]. In the present paper we give more details of this calculation and compare the result with the corresponding six-gluon MHV amplitude.

\subsection{Duality relation}

In this subsection we formulate and discuss the main point of the present paper - the proposed duality relation between the MHV planar amplitudes $\mathcal{M}_{n}^{(\mathrm{MHV})}$ and the light-like Wilson loops $W\left(C_{n}\right)$.

The conjectured duality states that in the planar $\mathcal{N}=4 \mathrm{SYM}$ theory the finite parts of the logarithms of the gluon amplitude and of the Wilson loop are equal (up to an inessential additive constant):

$$
F_{n}^{(\mathrm{MHV})}=F_{n}^{(\mathrm{WL})}+\text { const },
$$

upon the formal identification of the external on-shell gluon momenta in the amplitude with the light-like segments forming the closed polygon $C_{n}$ (the contour of the Wilson loop),

$$
p_{i}^{\mu}:=x_{i}^{\mu}-x_{i+1}^{\mu}
$$


Thus, the Mandelstam variables for the scattering amplitudes $t_{i}^{[j]}=\left(p_{i}+\ldots+p_{i+j-1}\right)^{2}$ are related to the distances $x_{i j}^{2}$ between two cusp points on the integration contour of $W\left(C_{n}\right)$ as follows,

$$
t_{i}^{[j]} / t_{k}^{[l]}:=x_{i, i+j}^{2} / x_{k, k+l}^{2} .
$$

The divergent parts of the scattering amplitudes and the light-like Wilson loops are also related to each other but the relationship is more subtle since the two objects are defined in two different schemes (infrared regularization for the amplitudes and ultraviolet regularization for the Wilson loops), both based on dimensional regularization. From the discussion in Subsections 2.2 and 2.3 we know that the leading IR divergence of the amplitude (the coefficient of the double pole $\epsilon_{\mathrm{IR}}^{-2}$ in Eq. (10) ) coincides with the leading UV divergence of the Wilson loop (the coefficient of the double pole $\epsilon_{\mathrm{UV}}^{-2}$ in Eq. (21)), since both are controlled by the universal cusp anomalous dimension $\Gamma_{\text {cusp }}(a)$. One can also achieve the matching of the coefficients of the subleading simple poles corresponding to the (non-universal) collinear anomalous dimensions $G(a)$ and $\Gamma(a)$ from Eqs. (12) and (22), respectively $3^{3}$ To this end one relates the parameters of the two different renormalization schemes as follows,

$$
x_{i, i+2}^{2} \mu_{\mathrm{UV}}^{2}:=t_{i}^{[2]} / \mu_{\mathrm{IR}}^{2} \mathrm{e}^{\gamma(a)}, \quad \quad \epsilon_{\mathrm{UV}}:=-\epsilon_{\mathrm{IR}} \mathrm{e}^{\epsilon_{\mathrm{IR}} \delta(a)} .
$$

Here the functions $\gamma(a)$ and $\delta(a)$ are chosen in a way to compensate the mismatch between $G(a)$ and $\Gamma(a)$, without creating extra $\mu$-dependent finite terms. It is easy to check that these functions are the solutions to the equations

$$
\begin{gathered}
\gamma(a) \Gamma_{\text {cusp }}(a)+\delta(a) \tilde{\Gamma}_{\text {cusp }}(a)+G(a)+\Gamma(a)=0, \\
\gamma(a) \tilde{\Gamma}_{\text {cusp }}(a)+2 \delta(a) \tilde{\tilde{\Gamma}}_{\text {cusp }}(a)+\tilde{G}(a)+\tilde{\Gamma}(a)=0,
\end{gathered}
$$

where $\tilde{\Gamma}_{\text {cusp }}(a)=\int_{0}^{a} \frac{d a^{\prime}}{a^{\prime}} \Gamma_{\text {cusp }}\left(a^{\prime}\right)$ and $\tilde{\tilde{\Gamma}}_{\text {cusp }}(a)=\int_{0}^{a} \frac{d a^{\prime}}{a^{\prime}} \tilde{\Gamma}_{\text {cusp }}\left(a^{\prime}\right)$, and similarly for $\tilde{G}(a)$ and $\tilde{\Gamma}(a)$. It should be stressed that this procedure is not analogous to comparing two different renormalization schemes for the computation of the same divergent object. It is rather a change of variables (regularization parameters) which allows us to compare two different objects computed in two different schemes. Another such change of variables is the identification (27) of the particle momenta with the light-like segments on the Wilson loop contour.

The duality relation (26) was inspired by the prescription of Alday and Maldacena for computing gluon scattering amplitudes at strong coupling [14], which essentially recasts the amplitudes into light-like Wilson loops in the dual variables (27). A priori, one would expect the strong coupling relation between gluon amplitudes and Wilson loops to receive $1 / \sqrt{\lambda}$ corrections, which might spoil the relation at weak coupling [14]. Nevertheless, in [17] three of us found that at one loop and for four points the Wilson loop and the gluon amplitude agree, which lead to the idea that the duality might also be true perturbatively. In [18] the duality at one loop was shown to apply also to $n$-point amplitudes. Further evidence in favor of the duality was accumulated by the present authors by two-loop calculations of the four-point [20] and five-point [21] Wilson loops, which were shown to match the corresponding gluon amplitudes according to Eqs. (26) and (27).

\footnotetext{
${ }^{3}$ We thank Paul Heslop for turning our attention to the incomplete discussion of this point in the first version of the present paper. We are also grateful to Lance Dixon for a discussion of the different physical interpretations of the IR and UV simple poles [49].
} 
What can we say about the proposed duality to all orders in the coupling $a$ ? Supposing the duality holds, one immediate consequence is that $F_{n}^{(\mathrm{MHV})}$, after making the change of variables (27), should satisfy the conformal Ward identity (24). We stress the fact that, while the conformal properties of the Wilson loop are manifest, there is no obvious reason to expect that the matching gluon amplitudes should have any reasonable behavior under conformal transformations acting on the particle momenta. Yet, the BDS ansatz for the MHV amplitudes does have this unexpected conformal property. For instance, when rewritten in terms of the dual variables $x_{i}$ (27), the BDS ansatz for four (14) and five (15) gluons reads as follows:

$$
\begin{aligned}
& F_{4}^{(\mathrm{BDS})}=\frac{1}{4} \Gamma_{\text {cusp }}(a) \ln ^{2}\left(\frac{x_{13}^{2}}{x_{24}^{2}}\right)+\text { const }, \\
& F_{5}^{(\mathrm{BDS})}=-\frac{1}{8} \Gamma_{\text {cusp }}(a) \sum_{i=1}^{5} \ln \left(\frac{x_{i, i+2}^{2}}{x_{i, i+3}^{2}}\right) \ln \left(\frac{x_{i+1, i+3}^{2}}{x_{i+2, i+4}^{2}}\right)+\text { const } .
\end{aligned}
$$

It is easy to check that these formulae are indeed solutions of the Ward identity (24). In fact, they are the unique solutions (up to an additive constant) for $n=4,5$, so they give the all-order form for $F_{4}^{(\mathrm{WL})}$ and $F_{5}^{(\mathrm{WL})}$. In particular, this confirms the duality up to three loops for $n=4$, since on the one hand, the BDS ansatz is known to be correct up to three loops in this case, and on the other hand, the $n=4$ Wilson loop does satisfy the Ward identity to all orders.

A hint at a possible source of this surprising conformal symmetry of the MHV amplitudes comes from the observation that all momentum integrals entering in the expression for $\mathcal{M}_{4}$ up to four [5, 39] (and possibly even five [50]) loops are of the 'pseudo-conformal' (also referred to as 'dual conformal') type [51, 17] 4] The conformal properties of such momentum integrals are revealed by rewriting them in terms of 'dual coordinates' according to (27). If their external legs are taken off shell, one can remove the dimensional regulator and the integrals become manifestly covariant under the action of the conformal group $S O(2,4)$ on the dual coordinates (i.e., on the particle momenta). It should be made very clear that this symmetry of the integrals (broken on shell by the IR divergencies) is not related to the original conformal symmetry of the $\mathcal{N}=4 \mathrm{SYM}$ theory, acting on the gluon fields in the configuration space. It is also important to realize that this unexpected property of the momentum integrals contributing to the MHV gluon amplitudes does not automatically imply that the amplitude should satisfy the anomalous (dual) conformal Ward identity (24). This is only true for some very special combinations of such pseudo-conformal integrals (for example, all the integrals in the four-gluon amplitude up to four (five) loops appear with coefficients \pm 1 [39, 50, 17, 52]). The exact link between the pseudo-conformal property of the integrals and the dual conformal behavior of the amplitude is still unclear.

At this stage one might suspect that the observed duality relation for $n=4,5$ is true only because both objects have the same conformal symmetry. To put it differently, the proposed duality might be reduced to the weaker (but still highly non-trivial) statement that the MHV gluon amplitudes have dual conformal symmetry. The first real test of the stronger form of the duality (26) is provided by the case $n=6$.

\footnotetext{
${ }^{4}$ The conformal properties of ladder (scalar box) multiloop integrals were first described by Broadhurst (see the first reference in [51]).
} 
The BDS ansatz for the six-gluon amplitude (16), rewritten in the dual variables $x_{i}$, reads

$$
\begin{aligned}
F_{6}^{(\mathrm{BDS})}=\frac{1}{4} \Gamma_{\text {cusp }}(a) \sum_{i=1}^{6} & {\left[-\ln \left(\frac{x_{i, i+2}^{2}}{x_{i, i+3}^{2}}\right) \ln \left(\frac{x_{i+1, i+3}^{2}}{x_{i, i+3}^{2}}\right)\right.} \\
+ & \left.\frac{1}{4} \ln ^{2}\left(\frac{x_{i, i+3}^{2}}{x_{i+1, i+4}^{2}}\right)-\frac{1}{2} \operatorname{Li}_{2}\left(1-\frac{x_{i, i+2}^{2} x_{i+3, i+5}^{2}}{x_{i, i+3}^{2} x_{i+2, i+5}^{2}}\right)\right]+ \text { const . }
\end{aligned}
$$

Again, we can immediately verify that this is a solution of the Ward identity (24). However, unlike the cases $n=4,5$ where the solutions (31), (32) are unique up to an additive constant, this is no longer true for $n \geq 6$. As explained at the end of Subsection 2.3. for $n \geq 6$ the general solution of (24) can contain an arbitrary function of the conformal cross-ratios (and of the coupling constant). Thus, for $n=6$, we can expect a possible deviation from the BDS ansatz of the form

$$
F_{6}^{(\mathrm{WL})}=F_{6}^{(\mathrm{BDS})}+R_{6}\left(u_{1}, u_{2}, u_{3} ; a\right) .
$$

The one-loop Wilson loop calculation of [18] has shown that the 'remainder' function $R_{6}$ is just a constant at one loop, which is a confirmation of the Wilson loop/scattering amplitude duality going beyond the scope of conformal symmetry. However, one might suspect just a low loop-order 'accident'. The point is that the function $R_{6}$ must satisfy a further, rather powerful constraint, the so-called collinear limit [2, 6] (see also Subsection 4.1 for further comments). It could be that due to the limited choice of loop integrals at this low perturbative level, the function (33) made of them is the only one satisfying both the conformal Ward identity (24) and the collinear limit. If so, at some higher perturbative level new functions with these properties might appear which could spoil the BDS ansatz and/or the Wilson loop/scattering amplitude duality.

Indeed, our recent explicit $n=6$ Wilson loop calculation 24 has shown that at two loops there exists a non-trivial 'remainder' function $R_{6}$ satisfying both conditions. The crucial test then was to compare the results of our Wilson loop calculation with a parallel two-loop six-gluon amplitude calculation, in order to check whether the proposed duality between Wilson loops and gluon amplitudes continues to hold at this level (which, if true, automatically implies the breakdown of the BDS ansatz). The results of the six-gluon calculation have become available very recently [13]. As we demonstrate in Section 4, the detailed numerical comparisons of the two calculations shows that indeed the BDS ansatz fails at two loops whereas the duality with Wilson loops is preserved,

$$
F_{6}^{(\mathrm{MHV})}=F_{6}^{(\mathrm{WL})}-c_{6}(a) .
$$

We consider this very strong evidence that the duality should hold to all orders in the coupling, although the 'remainder' function $R_{n}$ is likely to receive corrections at each loop order.

\section{Light-like hexagon Wilson loop}

The two-loop calculation of the light-like hexagon Wilson loop $W\left(C_{6}\right)$ goes along the same lines as the analysis of the rectangular $(n=4)$ and pentagonal $(n=5)$ Wilson loops $W\left(C_{n}\right)$ performed in Refs. [20, 19], where the interested reader can find the details of the technique employed.

As was explained in detail in [20], the two-loop calculation of $W\left(C_{6}\right)$ can be significantly simplified by making use of the non-Abelian exponentiation property of Wilson loops [53]. In 
application to $W\left(C_{6}\right)$, it can be formulated as follows:

$$
\ln W\left(C_{6}\right)=\frac{g^{2}}{4 \pi^{2}} C_{F} w^{(1)}+\left(\frac{g^{2}}{4 \pi^{2}}\right)^{2} C_{F} N w^{(2)}+O\left(g^{6}\right),
$$

where $C_{F}=\left(N^{2}-1\right) /(2 N)$ is the quadratic Casimir of the $S U(N)$ in the fundamental representation and $w^{(1,2)}$ are dimensionless $\left(N\right.$-independent) functions of the distances $x_{i j}^{2}$ and UV cut-off $\mu_{\mathrm{UV}}^{2}$. In calculating $w^{(2)}$ we do not rely on the planar limit. As a result, equation (36) is exact in $N .5$ We will take the planar limit later on, when we need to compare our result to the planar MHV amplitude. The one-loop correction $w^{(1)}$ was found in [18]. Its divergent part coincides with the one-loop contribution to $Z_{n}$, Eq. (10), and its finite part coincides up to an additive constant with the BDS ansatz (13) and satisfies the duality relation (4) at one loop.

For finite $N$ the two-loop corrections to $W\left(C_{6}\right)$ involve two different color factors $C_{F}^{2}$ and $C_{F} N$. According to (36), the coefficient in front of $g^{4} C_{F}^{2} /\left(4 \pi^{2}\right)^{2}$ in the two-loop expression for $W\left(C_{6}\right)$ is given by $\left(w^{(1)}\right)^{2} / 2$. Therefore, in order to determine the function $w^{(2)}$ one has to calculate the contribution to $W\left(C_{6}\right)$ only from two-loop diagrams containing 'maximally nonAbelian' color factor $C_{F} N$. This property significantly reduces the number of relevant two-loop diagrams. The function $w^{(2)}$ is gauge invariant, so in order to simplify the calculation we shall employ the Feynman gauge. In this gauge, some of the 'maximally non-Abelian' diagrams like those where both ends of a gluon are attached to the same light-like segment vanish by virtue of $x_{j, j+1}^{2}=0$. The corresponding Feynman diagrams have the same topology as for the rectangular $(n=4)$ and pentagon $(n=5)$ Wilson loops $W\left(C_{n}\right)$ and they can easily be identified by applying the selection rules formulated in Ref. [20].

To summarize, in Fig. 2 we list all non-vanishing two-loop diagrams of different topologies contributing to $w^{(2)}$. The diagrams shown in Figs. 2(m) - (r) involve the three-gluon interaction vertex of the $\mathcal{N}=4 \mathrm{SYM}$ Lagrangian. Their color factors equal $C_{F} N$, and therefore they contribute to the function $w^{(2)}$ in (36). The non-planar diagrams in Figs. 2(a) - (1) involve two free gluon propagators and their color factors equal $C_{F}\left(C_{F}-N / 2\right)$. To identify their contribution to $w^{(2)}$, we have to retain the maximally non-Abelian part only, that is, to replace their color factors by $C_{F}\left(C_{F}-N / 2\right) \rightarrow-C_{F} N / 2$. Finally, the diagrams in Figs. 2(s) $-(\mathrm{u})$ involve the one-loop correction to the gluon propagator with the blob denoting gauge fields/gauginos/scalars/ghosts propagating along the loop. Their color factors equal $C_{F} N$ and they directly contribute to $w^{(2)}$. To preserve supersymmetry, we evaluate these diagrams within the dimensional reduction scheme. The two-loop correction $w^{(2)}$ is given by the sum over the individual diagrams shown in Fig. 2 plus crossing symmetric diagrams obtained either by cyclic permutations of the cusp points $(1,2, \ldots, n) \mapsto(n, 1, \ldots, n-1)$, or by flips $(1,2, \ldots, n) \mapsto(n, n-1, \ldots, 1)$ with $n=6$.

To compute the diagrams shown in Fig. 2, we employ the technique developed in Refs. [38, 19. The calculation goes along the same lines as for the rectangular and pentagonal Wilson loops [17, 20, 21] and the result can be summarized as follows. It is convenient to expand the contribution of each diagram in powers of $1 / \epsilon$ and separate the UV divergent and finite parts as

$$
w^{(2)}=\sum_{\alpha}\left\{\frac{1}{2}\left(\frac{1}{\epsilon^{4}} A_{-4}^{(\alpha)}+\frac{1}{\epsilon^{3}} A_{-3}^{(\alpha)}+\frac{1}{\epsilon^{2}} A_{-2}^{(\alpha)}+\frac{1}{\epsilon} A_{-1}^{(\alpha)}\right) \sum_{i=1}^{6}\left(-x_{i, i+2}^{2} \mu_{\mathrm{UV}}^{2}\right)^{2 \epsilon}+A_{0}^{(\alpha)}\right\}+O(\epsilon),
$$

\footnotetext{
${ }^{5} \mathrm{Up}$ to three loops, the color factors in front of $w^{(n)}$ on the right-hand side of (36) have the form $C_{F} N^{n}$ and the only source of non-planar corrections is the subleading term in the expression for the Casimir $C_{F}=N / 2-1 /(2 N)$. Starting from four loops, the color factors are not expressible in terms of simple Casimir operators and receive genuine nonplanar corrections $[53]$.
} 


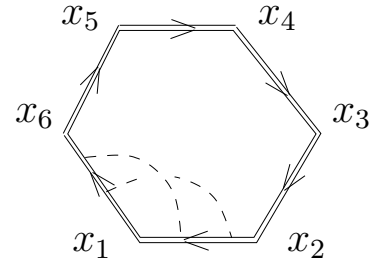

(a)

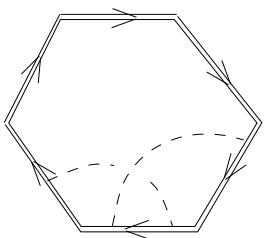

(e)

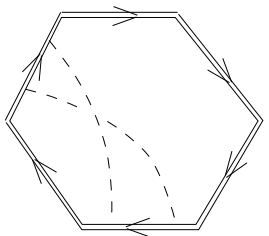

(i)



(m)

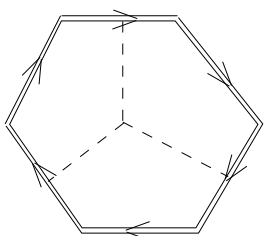

(q)

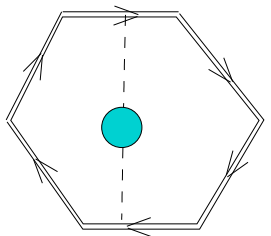

(u)

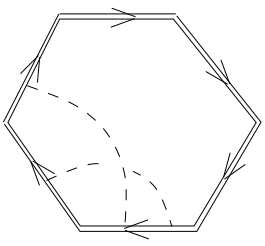

(b)

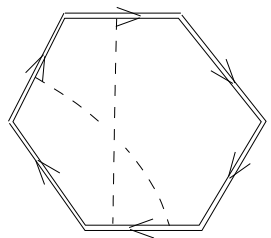

(f)

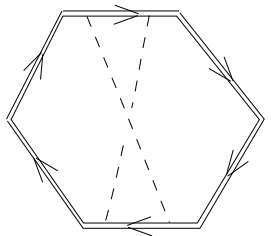

(j)

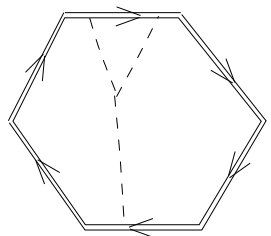

(n)

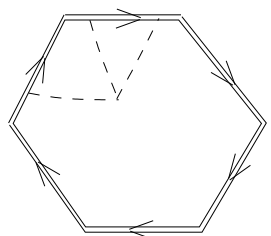

(r)

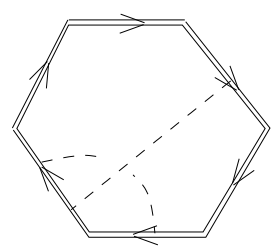

(c)

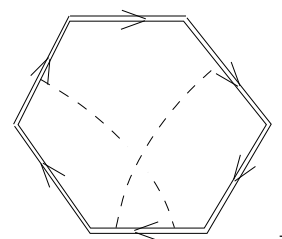

(g)

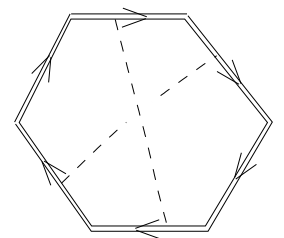

(k)

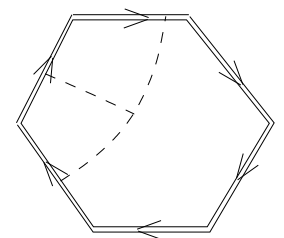

(o)

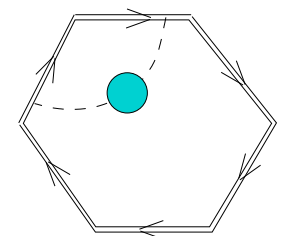

(s)

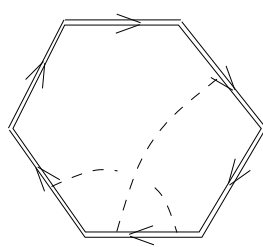

(d)

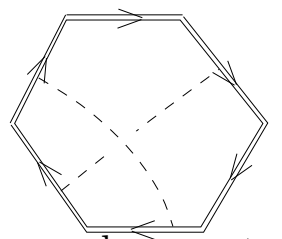

(h)

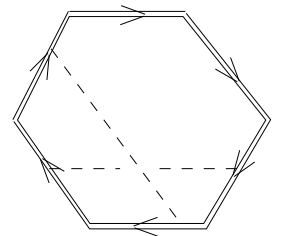

(l)

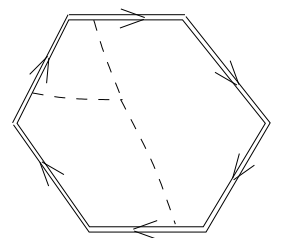

(p)

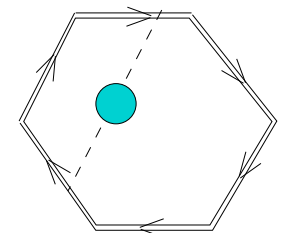

(t)

Figure 2: The maximally non-Abelian Feynman diagrams of different topology contributing to $F_{6}^{(\mathrm{WL})}$. The double lines depict the integration contour $C_{6}$, the dashed lines the gluon propagator and the blob the one-loop polarization operator. 
where $\epsilon=\epsilon_{\mathrm{UV}}$ and the scale $\mu_{\mathrm{UV}}^{2}$ is defined in (23). Here the index $\alpha$ runs over the twoloop Feynman diagrams shown in Fig. 2(a)-(u) and the factor 1/2 has been inserted for later convenience. For each diagram, the coefficient functions $A_{-n}^{(\alpha)}$ depend on dimensionless ratios of distances $x_{i, i+2}^{2}$ and $x_{i, i+3}^{2}$ (with $i=1, \ldots, 6$ and the periodicity condition $i+6 \equiv i$ ). We would like to stress that the contribution of each individual diagram to $w^{(2)}$, or equivalently the functions $A_{-n}^{(\alpha)}$, are gauge dependent and it is only their sum on the right-hand side of (37) that remains gauge invariant. We note that many of the coefficient functions $A_{-n}^{(\alpha)}$ can be cross-checked against similar contributions to $W\left(C_{5}\right)$ and $W\left(C_{4}\right)$ [20, 21], which can be obtained by shrinking one and two segments, respectively, of the contour $C_{6}$ to a point.

\subsection{Divergent part}

Summarizing our results for the UV divergent part of $W\left(C_{6}\right)$, we find that the coefficients $A_{-4}^{(\alpha)}$, $A_{-3}^{(\alpha)}$ and $A_{-2}^{(\alpha)}$ of the poles on the right-hand side of (37) are given by:

- $O\left(\epsilon^{-4}\right)$ terms only come from the two Feynman diagrams shown in Figs. 2(a) and 2 (r).

$$
A_{-4}^{(\mathrm{a})}=-\frac{1}{16}, \quad A_{-4}^{(\mathrm{r})}=\frac{1}{16}
$$

- $O\left(\epsilon^{-3}\right)$ terms only come from the two Feynman diagrams shown in Figs. 2(r) and 2(s).

$$
A_{-3}^{(\mathrm{r})}=-\frac{1}{8}, \quad A_{-3}^{(\mathrm{s})}=\frac{1}{8}
$$

- $O\left(\epsilon^{-2}\right)$ terms only come from the Feynman diagrams shown in Figs. 2(a), (e), (o), (r) and (s) .

$$
A_{-2}^{(\mathrm{a})}=-\frac{\pi^{2}}{96}, \quad A_{-2}^{(\mathrm{e})}=-\frac{\pi^{2}}{24}, \quad A_{-2}^{(\mathrm{o})}=\frac{\pi^{2}}{48}, \quad A_{-2}^{(\mathrm{r})}=-\frac{1}{4}+\frac{5}{96} \pi^{2}, \quad A_{-2}^{(\mathrm{s})}=\frac{1}{4}
$$

All these contributions match the corresponding ones for $W\left(C_{4}\right)$ and $W\left(C_{5}\right)$.

- $O\left(\epsilon^{-1}\right)$ terms come from the Feynman diagrams shown in Figs. 2(a)-2(e) $2(\mathrm{~m})-2(\mathrm{p})$ and 2(r) - 2(u).

The expressions for $A_{-1}^{(\mathrm{a})}, A_{-1}^{(\mathrm{e})}, A_{-1}^{(\mathrm{o})}$ and $A_{-1}^{(\mathrm{s})}$ are the same as for $W\left(C_{4}\right)$ and $W\left(C_{5}\right)$, while the remaining $A_{-1}$-coefficients are given by complicated functions of the distances $x_{i, i+2}^{2}$ and $x_{i, i+3}^{2}$. To determine them we apply the 'subtraction procedure' described in [21]. As we will see shortly, these functions cancel against each other in the sum of all diagrams leading to

$$
\sum_{\alpha} A_{-1}^{(\alpha)}=\frac{7}{8} \zeta_{3}
$$

Substituting the relations (38) - (41) into (37) we find that the divergent part of $w^{(2)}$ is given by

$$
w^{(2)}=\left\{\epsilon^{-2} \frac{\pi^{2}}{96}+\epsilon^{-1} \frac{7}{16} \zeta_{3}\right\} \sum_{i=1}^{6}\left(-x_{i, i+2}^{2} \mu^{2}\right)^{2 \epsilon}+O\left(\epsilon^{0}\right) .
$$




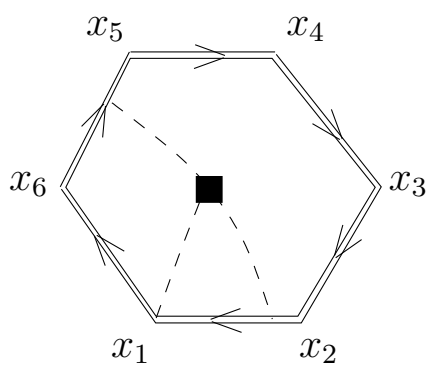

(a)

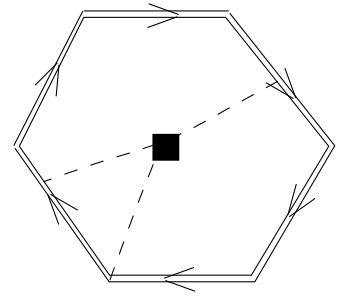

(b)

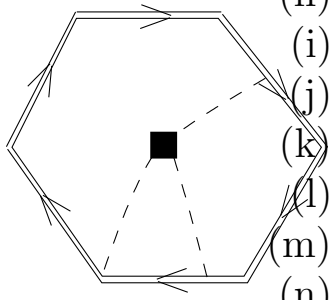

(c)

Figure 3: The auxiliary Feynman diagrams defined in (44). The double line depicts the integration contour $C_{6}$, the dashed line the gluon propagator and the box the fictitious three-gluon vertex (45).

As we have shown in [21], a similar relation holds for an arbitrary $n$-gon light-like Wilson loop $W\left(C_{n}\right)$ (with $n \geq 4$ ).

In the planar limit, we take into account that $C_{F}=N / 2$ and rewrite (36) as

$$
\ln W\left(C_{6}\right)=a w^{(1)}+2 a^{2} w^{(2)}+O\left(a^{3}\right) .
$$

Combining it with (42) we see that the divergent part of $\ln W\left(C_{6}\right)$ is of the expected form (21).

Let us now return to (41) and specify the contribution of individual diagrams. We introduce, following [21, the auxiliary Feynman diagrams shown in Fig. 3, These diagrams involve a new fake 'interaction vertex' for three gluons. Notice that one of the gluons in Fig. 3 is attached to a corner of the hexagon, while the positions of the two remaining gluons are integrated over one adjacent segment and one non-adjacent segment. By definition [21], the Feynman integrals associated with the two diagrams shown in Fig. 3 are

$$
\begin{aligned}
I_{\text {aux }}^{(\mathrm{a})} & =\frac{1}{2} g^{4} C_{F} N\left(x_{12} \cdot x_{56}\right) \int_{0}^{1} d \tau_{1} \int_{0}^{1} d \tau_{5} J\left(x_{1}, x_{2}+\tau_{1} x_{12}, x_{6}+\tau_{5} x_{56}\right), \\
I_{\text {aux }}^{(\mathrm{b})} & =\frac{1}{2} g^{4} C_{F} N\left(x_{61} \cdot x_{34}\right) \int_{0}^{1} d \tau_{3} \int_{0}^{1} d \tau_{6} J\left(x_{1}+\tau_{1} x_{61}, x_{1}, x_{4}+\tau_{3} x_{34}\right), \\
I_{\text {aux }}^{(\mathrm{c})} & =\frac{1}{2} g^{4} C_{F} N\left(x_{12} \cdot x_{34}\right) \int_{0}^{1} d \tau_{1} \int_{0}^{1} d \tau_{3} J\left(x_{1}, x_{2}+\tau_{1} x_{12}, x_{4}+\tau_{3} x_{34}\right),
\end{aligned}
$$

where we have used the parameterization (18) of the integration contour $C_{6}$ and have introduced the notation for the auxiliary function [9, 21]

$$
J\left(z_{1}, z_{2}, z_{3}\right)=-i\left(\mu_{\mathrm{UV}}^{2}\right)^{-\epsilon} \int d^{4-2 \epsilon} z G\left(z-z_{1}\right) G\left(z-z_{2}\right) G\left(z-z_{3}\right),
$$

with the gluon propagator $G(x)$ defined in (A.3). $J\left(z_{1}, z_{2}, z_{3}\right)$ is a symmetric function of the three points $z_{i}^{\mu}$ (with $i=1,2,3$ ) in Minkowski space-time.

We observe that on the right-hand side of (44) two of the points, say $z_{2}$ and $z_{3}$, are separated by a light-like interval, $z_{23}^{2} \equiv\left(z_{2}-z_{3}\right)^{2}=0$. In this case, $J\left(z_{1}, z_{2}, z_{3}\right)$ develops a simple pole in $\epsilon$ (see Eq. (A.8) for the explicit expression). It originates from the integration in (45) over $z^{\mu}$ approaching the light-like direction defined by the vector $\left(z_{2}-z_{3}\right)^{\mu}$, so that the distances $\left(z-z_{2}\right)^{2}$ and $\left(z-z_{3}\right)^{2}$ vanish simultaneously and the two propagators on the right-hand side of (45) become singular. As a consequence, the integrals $I_{\text {aux }}^{(\mathrm{a}, \mathrm{b}, \mathrm{c})}$ have a simple pole in $\epsilon$. Then, 
we add together the crossing symmetric diagrams of the same topology as in Fig. 3 and expand their contributions in $\epsilon$ similar to (37),

$$
I_{\text {aux }}^{(\alpha)}+(\text { cross-symmetry })=\left(\frac{g^{2}}{4 \pi^{2}}\right)^{2} C_{F} N\left[\frac{1}{2 \epsilon} M_{-1}^{(\alpha)} \sum_{i=1}^{6}\left(-x_{i, i+2}^{2} \mu_{\mathrm{UV}}^{2}\right)^{2 \epsilon}+M_{0}^{(\alpha)}+O(\epsilon)\right]
$$

with $\alpha=\{a, b, c\}$. Here $M_{-1}^{(\alpha)}$ and $M_{0}^{(\alpha)}$ are complicated functions of the distances $x_{j k}^{2}$. We will not need their explicit form for our purposes.

The crucial observation made in [21] is that the coefficient functions $A_{-1}^{(\alpha)}$ corresponding to the various diagrams shown in Fig. 2 can be expressed in terms of the three functions $M_{-1}^{(\mathrm{a})}$, $M_{-1}^{(\mathrm{b})}$ and $M_{-1}^{(\mathrm{c})}$. In Appendix A we illustrate the underlying mechanism by presenting a detailed calculation of the diagram shown in Fig. 2(c). Repeating the similar analysis for the remaining diagrams, we obtained the following expressions for the coefficient functions $A_{-1}^{(\alpha)}$ :

$$
\begin{array}{llrl}
A_{-1}^{(\mathrm{a})} & =-\frac{1}{24} \zeta_{3}, & A_{-1}^{(\mathrm{b})}=2 M_{-1}^{(\mathrm{a})}, & A_{-1}^{(\mathrm{c})}=2 M_{-1}^{(\mathrm{b})}, \\
A_{-1}^{(\mathrm{d})}=2 M_{-1}^{(\mathrm{c})}, & A_{-1}^{(\mathrm{e})}=\frac{1}{2} \zeta_{3}, & A_{-1}^{(\mathrm{m})}+A_{-1}^{(\mathrm{t})}=-M_{-1}^{(\mathrm{a})}-M_{-1}^{(\mathrm{c})}, \\
A_{-1}^{(\mathrm{n})}+A_{-1}^{(\mathrm{u})}=-M_{-1}^{(\mathrm{b})}, & A_{-1}^{(\mathrm{o})}=-M_{-1}^{(\mathrm{a})}+\frac{1}{8} \zeta_{3}, & A_{-1}^{(\mathrm{p})}=-M_{-1}^{(\mathrm{b})}-M_{-1}^{(\mathrm{c})}, \\
A_{-1}^{(\mathrm{r})}+A_{-1}^{(\mathrm{s})}=\frac{7}{24} \zeta_{3} . & &
\end{array}
$$

The functions $M_{-1}^{(\mathrm{a})}, M_{-1}^{(\mathrm{b})}$ and $M_{-1}^{(\mathrm{c})}$ can be expressed in terms of polylogarithms of degree 3 . Assigning degree 3 to the constant $\zeta_{3}$, we can say that the expressions appearing in (47) have the same degree of 'transcendentality' 6 . Adding them together we find that the functions $M_{-1}^{(\mathrm{a})}$, $M_{-1}^{(\mathrm{b})}$ and $M_{-1}^{(\mathrm{c})}$ cancel in the sum of all diagrams and we recover the relation (41).

To summarize, we have demonstrated by an explicit two-loop calculation that the divergent part of the hexagon Wilson loop has the expected form (21) with the cusp and collinear anomalous dimensions given by (12) and (22), respectively.

\section{$3.2 \quad$ Finite part}

The finite part of $W\left(C_{6}\right)$ receives contributions from all Feynman diagrams shown in Fig. 2. Substituting (37) into (36) and comparing with (20), we find the two-loop contribution to $F_{6}^{(\mathrm{WL})}=$ $\sum_{p \geq 1} a^{p} F_{6 ; p}^{(\mathrm{WL})}$

$$
F_{6 ; 2}^{(\mathrm{WL})}=2 \sum_{\alpha=\mathrm{a}, \ldots, \mathrm{u}} A_{0}^{(\alpha)}
$$

where the overall factor 2 comes from $\left(g^{2} /\left(4 \pi^{2}\right)\right)^{2} C_{F} N=2 a^{2}$ at large $N$. Based on our analysis of the rectangular and pentagon Wilson loops [20], we expect that the sum on the right-hand side of this relation should have degree of transcendentality 4.

As was already explained, the contribution of the diagrams shown in Figs. 2(a), (e), (r) and (s) to the finite part of $\ln W\left(C_{6}\right)$ can be obtained from the similar expressions for the rectangular

\footnotetext{
${ }^{6}$ Here we follow the nomenclature which has become common in the literature after the proposal of [54].
} 
Wilson loop [20],

$$
\begin{aligned}
& A_{0}^{(\mathrm{a})}=-\frac{6}{4} \cdot \frac{7}{2880} \pi^{4}, \\
& A_{0}^{(\mathrm{r})}+A_{0}^{(\mathrm{s})}=\frac{6}{4} \cdot \frac{119}{2880} \pi^{4}, \\
& A_{0}^{(\mathrm{e})}=\frac{\pi^{2}}{96} \sum_{i=1}^{6} \ln ^{2}\left(\frac{x_{i+1, i-1}^{2}}{x_{i, i-2}^{2}}\right)-\frac{6}{4} \cdot \frac{19}{720} \pi^{4},
\end{aligned}
$$

where the combinatorial factor $\frac{6}{4}$ accounts for the difference in the number of diagrams of the same topology between the hexagonal and rectangular Wilson loops. Moreover, the contribution of the diagrams shown in Fig. $2(\mathrm{~h}),(\mathrm{k})$ and (l) factorizes into a product of two one-loop integrals and can easily be calculated.

Let us separate the remaining diagrams into two groups according to their behavior as $\epsilon \rightarrow 0$ :

- The diagrams shown in Figs. 2(f), (g), (i), (j), (q) are finite;

- The diagrams shown in Figs. $2(\mathrm{~b}),(\mathrm{c}),(\mathrm{d}),(\mathrm{m}),(\mathrm{n}),(\mathrm{p}),(\mathrm{t}),(\mathrm{u})$ have a simple pole and the diagram in Fig. 2(o) has a double pole.

For the second group of diagrams we identify their finite part by employing the 'subtraction procedure' [21]. This amounts to subtracting from these diagrams the auxiliary diagrams shown in Fig. 3 with the appropriate weights defined by the coefficients in front of the functions $M_{-1}^{(\mathrm{a}, \mathrm{b}, \mathrm{c})}$ in (47). In this way, we compensate the simple poles in the above mentioned diagrams and render their contribution finite (with the only exception of the diagram in Fig. 2(o), where an additional subtraction of the double pole defined in (40) is required). Since the auxiliary diagrams also generate a finite part $M_{0}^{(\mathrm{a}, \mathrm{b}, \mathrm{c})}$, Eq. (46) , the subtractions will modify the finite parts of the individual diagrams, $A_{0}^{(\alpha)} \rightarrow \widehat{A}_{0}^{(\alpha)}$,

$$
\begin{array}{lll}
\widehat{A}_{0}^{(\mathrm{b})}=A_{0}^{(\mathrm{b})}-2 M_{0}^{(\mathrm{a})}, & \widehat{A}_{0}^{(\mathrm{c})}=A_{0}^{(\mathrm{c})}-2 M_{0}^{(\mathrm{b})}, & \widehat{A}_{0}^{(\mathrm{d})}=A_{0}^{(\mathrm{d})}-2 M_{0}^{(\mathrm{c})}, \\
\widehat{A}_{0}^{(\mathrm{n})+(\mathrm{u})}=A_{0}^{(\mathrm{n})}+A_{0}^{(\mathrm{u})}+M_{0}^{(\mathrm{b})}, & \widehat{A}_{0}^{(\mathrm{o})}=A_{0}^{(\mathrm{o})}+M_{0}^{(\mathrm{a})}, & \widehat{A}_{0}^{(\mathrm{p})}=A_{0}^{(\mathrm{p})}+M_{0}^{(\mathrm{b})}+M_{0}^{(\mathrm{c})}, \\
\widehat{A}_{0}^{(\mathrm{m})+(\mathrm{t})}=A_{0}^{(\mathrm{m})}+A_{0}^{(\mathrm{t})}+M_{0}^{(\mathrm{a})}+M_{0}^{(\mathrm{c})} . &
\end{array}
$$

This, however, does not affect the total sum of diagrams,

$$
\sum_{\alpha} A_{0}^{(\alpha)}=\sum_{\alpha} \widehat{A}_{0}^{(\alpha)}
$$

By construction, the subtracted diagrams are free from UV divergences and, therefore, can be directly evaluated in $D=4$ dimensions. In this way, we find that,

$$
\widehat{A}_{0}^{(\mathrm{n})+(\mathrm{u})}=\widehat{A}_{0}^{(\mathrm{m})+(\mathrm{t})}=0 .
$$

In addition, we obtain a representation for the remaining functions $\widehat{A}_{0}^{(\mathrm{b}, \mathrm{c}, \mathrm{d}, \mathrm{o}, \mathrm{p})}$ and $A_{0}^{(\mathrm{f}, \mathrm{g}, \mathrm{i}, \mathrm{j}, \mathrm{q})}$ in the form of convergent multiple integrals. Being combined with the explicit expressions for the remaining functions $A_{0}^{(\mathrm{a}, \mathrm{h}, \mathrm{e}, \mathrm{k}, \mathrm{l}, \mathrm{r}, \mathrm{s})}$, they determine the two-loop correction to the finite part of the 
hexagon Wilson loop (48). Their explicit expressions are lengthy and we do not present them here to save space. As an example, we give the detailed calculation of $\widehat{A}_{0}^{(\mathrm{c})}$ in the Appendix.

It is straightforward to evaluate numerically the sum on the right-hand side of (48) for a given set of kinematical invariants,

$$
K=\left\{x_{13}^{2}, x_{14}^{2}, x_{15}^{2}, x_{24}^{2}, x_{25}^{2}, x_{35}^{2}, x_{26}^{2}, x_{36}^{2}, x_{46}^{2}\right\},
$$

defined as the distances between the vertices of the hexagon $C_{6}$. We should take into account, however, that in $D=4$ dimensions there exists a relationship between the distances (53), such that only eight of them are independent. This relation reflects the fact that the five fourdimensional vectors $p_{i}^{\mu}=x_{i}^{\mu}-x_{i+1}^{\mu}$ (with $\left.i=1, \ldots, 5\right)$ are linearly dependent, and hence their Gram determinant vanishes

$$
G[K]=\operatorname{det}\left\|\left(p_{i} \cdot p_{j}\right)\right\|=0, \quad(i, j=1, \ldots, 5) .
$$

Using $p_{i}=x_{i}-x_{i+1}$, the entries of the matrix can be written as linear combinations of the distances (53).

We would like to note that the sum on the right-hand side of (48) defines a function of $x_{i j}^{2}$ even for configurations $K$ which do not satisfy (54). This function can be viewed as a particular continuation of the finite part of the hexagon Wilson loop off the hypersurface defined by (54).

\section{Duality relation for the six-gluon MHV amplitude}

In the parallel publication [13], an impressive two-loop calculation of the six-gluon MHV planar amplitude in $\mathcal{N}=4 \mathrm{SYM}$ theory has been performed. To verify the duality relation (35) we shall compare our results for the finite part of the hexagon Wilson loop with the numerical results from Ref. [13].

\subsection{The hexagon Wilson loop versus the BDS ansatz}

Let us first consider the relation between the hexagon Wilson loop and the BDS ansatz, Eq. (34). To two-loop accuracy it takes the form

$$
R_{\mathrm{W}}\left(u_{1}, u_{2}, u_{3}\right)=F_{6 ; 2}^{(\mathrm{WL})}-F_{6 ; 2}^{(\mathrm{BDS})}
$$

where $R_{\mathrm{W}}$ and $F_{6 ; 2}^{(\mathrm{BDS})}$ denote the two-loop contributions to the remainder function (5) and to the BDS ansatz (13), respectively. We recall that the functions $F_{6 ; 2}^{(\mathrm{WL})}$ and $F_{6 ; 2}^{(\mathrm{BDS})}$ satisfy the Ward identity (24) and $R_{\mathrm{W}}$ is a function of the three conformal cross-ratios (25) only.

The simplest way to check relation (55) is to evaluate the difference $F_{6 ; 2}^{(\mathrm{WL})}-F_{6 ; 2}^{(\mathrm{BDS})}$ for two different kinematical configurations $K$ and $K^{\prime}$, related to each other by a conformal $S O(2,4)$ transformations of the coordinates $x_{i}^{\mu}$ (with $\left.i=1, \ldots, 6\right)$. Since $F_{6 ; 2}^{(\mathrm{WL})}$ and $F_{6 ; 2}^{(\mathrm{BDS})}$ are dimensionless functions of the distances $x_{i j}^{2}$, they are automatically invariant under translations, Lorentz rotations and dilatations of the coordinates $x_{i}^{\mu}$. The only non-trivial transformations are the special conformal transformations (boosts), which are combinations of an inversion, a translation and another inversion. 
Let us start with the kinematical configuration $K=K\left(x_{i j}^{2}\right)$, Eq. (53), and perform an inversion of the coordinates, $x_{i}^{\mu} \rightarrow x_{i}^{\mu} / x_{i}^{2}$, to define the new configuration

$$
K^{\prime}=K\left(x_{i j}^{2} /\left(x_{i}^{2} x_{j}^{2}\right)\right)
$$

Since the variables $u(25)$ are invariant under such transformations, $u_{a}[K]=u_{a}\left[K^{\prime}\right]$, the difference $F_{6 ; 2}^{(\mathrm{WL})}-F_{6 ; 2}^{(\mathrm{BDS})}$ should also be invariant.

As an example, let us consider six light-like four-dimensional vectors $p_{i}^{\mu}$,

$$
\begin{array}{lll}
p_{1}=(1,1,0,0), & p_{2}=(-1, p, p, 0), & p_{3}=(1,-p, p, 0), \\
p_{4}=(-1,-1,0,0), & p_{5}=(1,-p,-p, 0), & p_{6}=(-1, p,-p, 0),
\end{array}
$$

with $p=1 / \sqrt{2}$ and $\sum_{i} p_{i}^{\mu}=0$. These vectors define the external momenta of the gluons in the six-gluon amplitude. Applying the duality relation $p_{i}=x_{i}-x_{i+1}$, we evaluate the corresponding distances (표),

$$
\begin{aligned}
K^{(\mathrm{a})}: & x_{14}^{2}=x_{15}^{2}=x_{24}^{2}=x_{25}^{2}=-2, \quad x_{36}^{2}=-2-2 \sqrt{2} \\
x_{13}^{2} & =x_{35}^{2}=x_{26}^{2}=x_{46}^{2}=-2-\sqrt{2}
\end{aligned}
$$

and the conformal cross-ratios (25),

$$
u_{1}=u_{3}=\frac{1}{2}+\frac{1}{2} \sqrt{2}, \quad u_{2}=1 .
$$

By construction, this kinematical configuration satisfies the Gram determinant constraint (54). To define the conformal transformations (56), we choose an arbitrary reference four-vector $x_{1}^{\mu}=$ $\left(x_{1}^{0}, x_{1}^{1}, x_{1}^{2}, x_{1}^{3}\right)$ and reconstruct the remaining $x$-vectors according to $x_{i+1}^{\mu}=x_{i}^{\mu}-p_{i}^{\mu}$ (due to translation invariance, the Wilson loop does not depend on the choice of $\left.x_{1}^{\mu}\right)$. Then, relation (55) implies that the function $R_{\mathrm{W}}$ evaluated for the kinematical configuration (56) should be the same as for the original configuration $K$.

As an example, we choose $x_{1}^{\mu}=(1,1,1,1)$ and apply the conformal transformation (56) to $K^{(a)}$ defined in (58) to obtain the new kinematical configuration

$$
\begin{aligned}
K^{(\mathrm{b})}: & x_{14}^{2}=x_{15}^{2}=x_{24}^{2}=x_{25}^{2}=-\frac{1}{2}-\frac{1}{4} \sqrt{2}, \quad x_{36}^{2}=-1-\frac{3}{4} \sqrt{2} \\
x_{13}^{2} & =-\frac{3}{2}-\sqrt{2}, \quad x_{35}^{2}=-\frac{5}{2}-\frac{7}{4} \sqrt{2}, \quad x_{26}^{2}=-\frac{1}{4}-\frac{1}{8} \sqrt{2}, \\
x_{46}^{2} & =-\frac{3}{8}-\frac{1}{4} \sqrt{2} .
\end{aligned}
$$

The results of our numerical tests are summarized in Table 1. They clearly show that $F_{6 ; 2}^{(\mathrm{WL})}$ and $F_{6 ; 2}^{(\mathrm{BDS})}$ vary under conformal transformations whereas their difference $R_{\mathrm{W}}=F_{6 ; 2}^{(\mathrm{WL})}-F_{6 ; 2}^{(\mathrm{BDS})}$ stays invariant.

We recall that in four dimensions the kinematical invariants (53) have to verify the Gram determinant constraint (54). This relation $G[K]=0$ is invariant under the conformal transformations (56), simply because the conformal boosts map six light-like vectors $p_{i}^{\mu}$ into another set of light-like vectors. There exist, however, certain kinematical configurations $K^{\prime}$ for which $u_{i}[K]=u_{i}\left[K^{\prime}\right]$ but $G\left[K^{\prime}\right] \neq 0$. Since the difference function $R_{\mathrm{W}}$ only depends on the $u$-variables, its value should be insensitive to the Gram determinant condition 7 . For example, consider the

\footnotetext{
${ }^{7}$ We recall that the functions entering (55) can be defined for configurations $K^{\prime}$ satisfying $G\left[K^{\prime}\right] \neq 0$.
} 
following kinematical configuration

$$
\begin{aligned}
K^{(\mathrm{c})}: \quad x_{14}^{2} & =x_{15}^{2}=-1, \quad x_{24}^{2}=x_{25}^{2}=-2, \quad x_{36}^{2}=-2-2 \sqrt{2}, \\
x_{13}^{2} & =-1-1 / \sqrt{2}, \quad x_{35}^{2}=x_{26}^{2}=x_{46}^{2}=-2-\sqrt{2} .
\end{aligned}
$$

The corresponding conformal cross-ratios (25) are given by (59), but $G\left[K^{(\mathrm{c})}\right] \neq 0$. We verified numerically that $R_{\mathrm{W}}\left[K^{(\mathrm{a})}\right]=R_{\mathrm{W}}\left[K^{(\mathrm{b})}\right]=R_{\mathrm{W}}\left[K^{(\mathrm{c})}\right]$ with accuracy $<10^{-5}$ (see Table 1). This observation allows us to study the function $R_{\mathrm{W}}\left(u_{1}, u_{2}, u_{3}\right)$ without any reference to the Gram determinant condition.

\begin{tabular}{||c||c|c|c||}
\hline \hline Kinematical point & $F_{6 ; 2}^{(\mathrm{WL})}$ & $F_{6 ; 2}^{(\mathrm{BDS})}$ & $R_{\mathrm{W}}$ \\
\hline \hline$K^{(\mathrm{a})}$ & -5.014825 & -14.294864 & 9.280039 \\
\hline$K^{(\mathrm{b})}$ & -6.414907 & -15.694947 & 9.280040 \\
\hline$K^{(\mathrm{c})}$ & -5.714868 & -14.994906 & 9.280038 \\
\hline
\end{tabular}

Table 1: Two-loop contributions to the hexagon Wilson loop, $F_{6 ; 2}^{(\mathrm{WL})}$, to the BDS ansatz, $F_{6 ; 2}^{(\mathrm{BDS})}$, and to their difference, $R_{\mathrm{W}}$, evaluated for three kinematical configurations (58), (60) and (61) corresponding to the same values of $u_{1}, u_{2}$ and $u_{3}$, Eq. (59).

The properties of the remainder function (55) were studied in our previous publication Ref. [24]. We found that $R_{\mathrm{W}}\left(u_{1}, u_{2}, u_{3}\right)$ is a completely symmetric function of $u_{i}$. For $u_{1}=0$, $u_{2}=u$ and $u_{3}=1-u$ its value is independent of $u$,

$$
R_{\mathrm{W}}(0, u, 1-u)=c_{\mathrm{W}} .
$$

As explained in [24], this property ensures that the hexagon Wilson loop has the same behavior as the gluon amplitude in the collinear limit. For the Wilson loop this corresponds to flattening one of the cusp points. This lends additional support to the duality relation between the Wilson loops and the scattering amplitudes. We found numerically that the value of $c_{\mathrm{W}}$ is given by

$$
c_{\mathrm{W}}=12.1756 \text {. }
$$

This number has a much smaller absolute accuracy $\left(\sim 10^{-3}\right)$ compared to the numbers in Table 1 because its evaluation requires taking differences of quantities that diverge in the collinear limit.

\subsection{The hexagon Wilson loop versus the six-gluon MHV amplitude}

We now compare numerically the function $R_{\mathrm{W}}$ defined in (55) with the analogous quantity defined for the MHV amplitude,

$$
R_{\mathrm{A}}=F_{6 ; 2}^{(\mathrm{MHV})}-F_{6 ; 2}^{(\mathrm{BDS})} .
$$

In order to test the duality relation (35), we have to show that for general kinematical configurations $K$ defined in equation (53),

$$
R_{\mathrm{W}}[K]-R_{\mathrm{A}}[K]=c_{\mathrm{W}} .
$$


Since $R_{\mathrm{W}}$ is a function of $u_{1}, u_{2}, u_{3}$ only, this relation would imply that so is $R_{\mathrm{A}}$.

To get rid of the constant $c_{\mathrm{W}}$, which, as we have seen, has lower numerical precision than the evaluations for generic kinematics, we subtract from (65) the same relation evaluated for some reference kinematical configuration $K^{(0)}$,

$$
R_{\mathrm{A}}[K]-R_{\mathrm{A}}\left[K^{(0)}\right]=R_{\mathrm{W}}[K]-R_{\mathrm{W}}\left[K^{(0)}\right] .
$$

The numerical tests of this relation for different kinematical configurations are summarized in Table 2.

\begin{tabular}{||c|c||c|c||}
\hline \hline Kinematical point & $\left(u_{1}, u_{2}, u_{3}\right)$ & $R_{\mathrm{W}}-R_{\mathrm{W}}^{(0)}$ & $R_{\mathrm{A}}-R_{\mathrm{A}}^{(0)}$ \\
\hline \hline$K^{(1)}$ & $(1 / 4,1 / 4,1 / 4)$ & $<10^{-5}$ & $-0.018 \pm 0.023$ \\
\hline$K^{(2)}$ & $(0.547253,0.203822,0.88127)$ & -2.75533 & $-2.753 \pm 0.015$ \\
\hline$K^{(3)}$ & $(28 / 17,16 / 5,112 / 85)$ & -4.74460 & $-4.7445 \pm 0.0075$ \\
\hline$K^{(4)}$ & $(1 / 9,1 / 9,1 / 9)$ & 4.09138 & $4.12 \pm 0.10$ \\
\hline$K^{(5)}$ & $(4 / 81,4 / 81,4 / 81)$ & 9.72553 & $10.00 \pm 0.50$ \\
\hline
\end{tabular}

Table 2: Comparison of the deviation from the BDS ansatz of the Wilson loop, $R_{\mathrm{W}}$, and of the six-gluon amplitude, $R_{\mathrm{A}}$, evaluated for the kinematical configurations (67). Here, $R_{\mathrm{W}}^{(0)}=$ $R_{\mathrm{W}}(1 / 4,1 / 4,1 / 4)=13.26530$ and $R_{\mathrm{A}}^{(0)}=R_{\mathrm{A}}(1 / 4,1 / 4,1 / 4)=1.0937 \pm 0.0057$ denote the same quantities evaluated at the reference kinematical point $K^{(0)}$. The numerical results for $R_{\mathrm{A}}$ and $R_{\mathrm{A}}^{(0)}$ are taken from Ref. [13.

$$
\begin{aligned}
K^{(0)}: & x_{i, i+2}^{2}=-1, \quad x_{i, i+3}^{2}=-2 ; \\
K^{(1)}: & x_{13}^{2}=-0.7236200, \quad x_{24}^{2}=-0.9213500, \quad x_{35}^{2}=-0.2723200, \quad x_{46}^{2}=-0.3582300, \\
& x_{15}^{2}=-0.4235500, \quad x_{26}^{2}=-0.3218573, \quad x_{14}^{2}=-2.1486192, \quad x_{25}^{2}=-0.7264904, \\
& x_{36}^{2}=-0.4825841 ; \\
K^{(2)}: & x_{13}^{2}=-0.3223100, \quad x_{24}^{2}=-0.2323220, \quad x_{35}^{2}=-0.5238300, \quad x_{46}^{2}=-0.8237640, \\
& x_{15}^{2}=-0.5323200, \quad x_{26}^{2}=-0.9237600, \quad x_{14}^{2}=-0.7322000, \quad x_{25}^{2}=-0.8286700, \\
& x_{36}^{2}=-0.6626116 ; \\
K^{(3)}: & x_{i, i+2}^{2}=-1, \quad x_{14}^{2}=-1 / 2, \quad x_{25}^{2}=-5 / 8, \quad x_{36}^{2}=-17 / 14 ; \\
K^{(4)}: & x_{i, i+2}^{2}=-1, \quad x_{i, i+3}^{2}=-3 ; \\
K^{(5)}: & x_{i, i+2}^{2}=-1, \quad x_{i, i+3}^{2}=-9 / 2 .
\end{aligned}
$$

Among the six configurations in equation (67) only the first four verify the Gram determinant condition (54). Also, the configurations $K^{(0)}$ and $K^{(1)}$ are related to each other by a conformal 
transformation. This explains why the first entry in the third column of Table 2 is almost zero, and also reflects the high precision of the numerical evaluation for the Wilson loop. Comparing the numerical values for the six-gluon amplitude and the hexagon Wilson loop, we observe that their finite parts coincide within the error bars. Therefore, we conclude that the duality relation (44) is satisfied, at least to two loops.

\section{Conclusions}

The results presented in this paper and in the parallel work [13] show that the conjectured Wilson loop/MHV amplitude duality holds at two loops for six cusp points/gluons, extending previous checks in a highly non-trivial way. We believe the evidence presented here indicates very strongly that the duality holds to all orders in the coupling. As pointed out in [24], this necessarily implies the failure of the BDS ansatz for six gluons and presumably beyond. Furthermore, the fact that the Wilson loop and the MHV amplitude both differ from the BDS ansatz by the same non-trivial function $R_{\mathrm{W}}\left(u_{1}, u_{2}, u_{3}\right)$ suggests that there is much more to the proposed duality than a dual conformal symmetry of the gluon amplitude. An issue which needs to be addressed in the near future is the analytical evaluation of the remainder function. It could give us clues about hidden symmetries of the problem and hints for generalizing it to higher loops, as well as to strong coupling.

While at strong coupling the duality naturally emerges from the gauge/string duality, at weak coupling we are still lacking the understanding of its origin. Since we expect that the duality applies only to planar amplitudes, this suggests that the best way to address it will not be the Lagrangian formulation of $\mathcal{N}=4 \mathrm{SYM}$. Rather, it is more likely to follow from some effective description of planar MHV amplitudes. A possible candidate might be the twistor approach proposed in [55].

Finally, let us return to the dual conformal symmetry of the gluon amplitudes. Assuming that the duality with Wilson loops holds, this symmetry follows from the ordinary conformal symmetry of the light-like Wilson loops in $\mathcal{N}=4 \mathrm{SYM}$. This does not explain, however, the origin of the duality itself. We believe that further understanding of the duality relation will come from a deeper investigation of the symmetries of gluon amplitudes. One might speculate that dual conformal symmetry is just part of a larger set of symmetries yet to be discovered. One possible way to uncover such symmetries might be the loop equations 8 [56, 37] both at weak and strong coupling [57, 58]. Were these symmetries powerful enough to determine the finite parts of the planar amplitudes to all loops, one would conclude that this sector of $\mathcal{N}=4 \mathrm{SYM}$ is exactly solvable.

\section{Acknowledgements}

We would like to thank Fernando Alday, Costas Bachas, Benjamin Basso, Iosif Bena, Alexander Gorsky, Paul Heslop, Bernard Julia, Yaron Oz and Arkady Tseytlin for stimulating discussions. We are particularly grateful to Zvi Bern, Lance Dixon, David Kosower, Radu Roiban, Marcus Spradlin, Christian Vergu and Anastasia Volovich for numerous valuable discussions and for

\footnotetext{
${ }^{8}$ We would like to thank A. Gorsky for pointing out to us the formal similarity of the anomalous Ward identity (24) with the loop equations.
} 
sharing their numerical results. This research was supported in part by the French Agence Nationale de la Recherche under grant ANR-06-BLAN-0142.

\section{A Appendix: Two loop corrections to the hexagon Wil- son loop}

In this Appendix we present some details of the Wilson loop calculation. It serves to illustrate both the technique employed and to outline the general structure of two-loop corrections to the finite part of the hexagon Wilson loop. We will examine one diagram in detail, while for the rest we will just summarize the relevant formulae.

As our detailed example, we consider the Feynman diagram shown in Fig. 2(c). It involves two gluon propagators and originates from the expansion of the path-ordered exponential (17) to fourth order in gauge fields. The end-points of the propagators are attached to three light-like segments $\left[x_{6}, x_{1}\right],\left[x_{1}, x_{2}\right]$ and $\left[x_{3}, x_{4}\right]$. It is convenient to parameterize the points belonging to the light-like segment $x^{\mu}\left(\tau_{j}\right) \in\left[x_{j}, x_{j+1}\right]$ in the following way

$$
x^{\mu}\left(\tau_{j}\right)=x_{j+1}^{\mu}+\tau_{j} x_{j, j+1}^{\mu}, \quad\left(0 \leq \tau_{j} \leq 1\right)
$$

with $x_{j, j+1}^{\mu}=x_{j}^{\mu}-x_{j+1}^{\mu}$ and $x_{j, j+1}^{2}=0$. Then the contribution of the diagram shown in Fig. 2(c) to the Wilson loop reads

$$
\begin{aligned}
& I^{c}=(i g)^{4} N^{-1} \operatorname{tr}\left[t^{a} t^{b} t^{a} t^{b}\right] \int_{0}^{1} d \tau_{1} x_{12}^{\mu_{1}} \int_{0}^{1} d \tau_{6} x_{61}^{\mu_{6}} G_{\mu_{1} \mu_{6}}\left(x_{12}\left(1-\tau_{1}\right)+x_{61} \tau_{6}\right) \\
& \times \int_{0}^{1} d \tau_{3} x_{34}^{\mu_{3}} \int_{0}^{\tau_{6}} d \tau_{6}^{\prime} x_{61}^{\nu_{6}} G_{\mu_{3} \nu_{6}}\left(x_{13}+x_{34}\left(1-\tau_{3}\right)+x_{61} \tau_{6}^{\prime}\right)
\end{aligned}
$$

where the condition $\tau_{6}^{\prime} \leq \tau_{6}$ is due to the ordering of the gluons along the path and $G_{\mu \nu}(x-y)$ is the gluon propagator. In our calculation we employ the Feynman gauge and use dimensional regularization with $D=4-2 \epsilon$ and $\epsilon>0$, so that the gluon propagator is given by

$$
G_{\mu \nu}(x)=g_{\mu \nu} G(x), \quad G(x)=-\frac{\Gamma(1-\epsilon)}{4 \pi^{2}}\left(-x^{2}+i 0\right)^{-1+\epsilon}\left(\mu^{2} \pi\right)^{\epsilon} .
$$

We would like to stress that the contribution of each individual Feynman diagram to the Wilson loop is gauge dependent whereas the sum of all two-loop diagrams shown in Fig. 2 is gauge invariant. As a first step, we evaluate the color factor of $I^{c}$

$$
N^{-1} \operatorname{tr}\left[t^{a} t^{b} t^{a} t^{b}\right]=C_{F}\left(C_{F}-\frac{1}{2} N\right),
$$

with $C_{F}=t^{a} t^{a}=\left(N^{2}-1\right) /(2 N)$ being the quadratic Casimir of $S U(N)$ in the fundamental representation, and verify that it involves the maximally non-Abelian part $\sim C_{F} N$. As explained in Sect. 3, it is only this part of $I^{c}$ which contributes to $\ln W\left(C_{6}\right)$. Then, we make use of the identities $x_{j, j+1}^{2}=0$ to simplify $\left[x_{12}\left(1-\tau_{1}\right)+x_{61} \tau_{6}\right]^{2}=2\left(x_{12} x_{61}\right)\left(1-\tau_{1}\right) \tau_{6}$ and similarly for the second propagator in (A.2). The integration over $\tau_{1}, \tau_{3}$ and $\tau_{6}$ can easily be performed leading to

$$
\begin{aligned}
& I_{\mathrm{NA}}^{c}=\left(\frac{g^{2}}{4 \pi^{2}}\right)^{2} C_{F} N \frac{\Gamma^{2}(1-\epsilon)}{8 \epsilon^{3}}\left(\pi \mu^{2}\right)^{2 \epsilon}\left(-x_{26}^{2}\right)^{\epsilon} \\
& \times \int_{0}^{1} d t\left(t^{\epsilon}-1\right) \frac{\left(x_{46}^{2}-x_{36}^{2}\right)-\left(x_{14}^{2}-x_{13}^{2}\right)}{\left(x_{46}^{2}-x_{36}^{2}\right) t+\left(x_{14}^{2}-x_{13}^{2}\right) \bar{t}}\left[\left(-x_{14}^{2} \bar{t}-x_{46}^{2} t\right)^{\epsilon}-\left(-x_{13}^{2} \bar{t}-x_{36}^{2} t\right)^{\epsilon}\right]
\end{aligned}
$$


with $t \equiv \tau_{6}^{\prime}$ and $\bar{t}=1-t$. Here the subscript indicates that we retained only the maximal non-Abelian part of $I^{c}$. We observe that $I_{\mathrm{NA}}^{c}$ has a single pole as $\epsilon \rightarrow 0$. It comes from the integration region $\tau_{1} \rightarrow 1$.

To separate the divergent and finite parts of $I_{\mathrm{NA}}^{c}$ we use the subtraction procedure described in detail in Ref. [21]. We introduce the auxiliary Feynman diagram shown in Fig. 33(b),

$$
I_{\text {aux }}^{(\mathrm{b})}=\frac{1}{2} g^{4} C_{F} N\left(x_{34} \cdot x_{61}\right) \int_{0}^{1} d \tau_{3} \int_{0}^{1} d \tau_{6} J\left(x_{1}+\tau_{6} x_{61}, x_{1}, x_{4}+\tau_{3} x_{34}\right),
$$

where $J\left(z_{1}, z_{2}, z_{3}\right)$ stands for three propagators joined at the same point $z$ which is integrated out,

$$
J\left(z_{1}, z_{2}, z_{3}\right)=-i\left(\mu^{2}\right)^{-\varepsilon} \int d^{D} z G\left(z-z_{1}\right) G\left(z-z_{2}\right) G\left(z-z_{3}\right) .
$$

The relation (A.6) involves this function evaluated for $z_{1}=x_{1}+\tau_{6} x_{61}, z_{2}=x_{1}$ and $z_{3}=x_{4}+\tau_{3} x_{34}$, so that the points $z_{1}$ and $z_{2}$ are separated by a light-like interval. For $\left(z_{1}-z_{2}\right)^{2}=0$ the calculation of $J\left(z_{1}, z_{2}, z_{3}\right)$ yields 9 ]

$$
J\left(z_{1}, z_{2}, z_{3}\right)=\left(\pi \mu^{2}\right)^{2 \varepsilon} \frac{\Gamma(1-2 \varepsilon)}{64 \pi^{4} \varepsilon} \int_{0}^{1} \frac{d \tau(\tau \bar{\tau})^{-\varepsilon}}{\left[-\left(\tau z_{13}+\bar{\tau} z_{23}\right)^{2}\right]^{1-2 \varepsilon}}
$$

with $\bar{\tau}=1-\tau$. We substitute this relation into (A.6), change the integration variable via $t=\tau_{6} \tau$ and expand $I_{\text {aux }}^{(\mathrm{b})}$ in powers of $\epsilon$ to find after some algebra,

$$
I_{\mathrm{aux}}^{(\mathrm{b})}=\left(\frac{g^{2}}{4 \pi^{2}}\right)^{2} \frac{C_{F} N}{16 \epsilon} \int_{0}^{1} d t \ln t \frac{\left(x_{46}^{2}-x_{36}^{2}\right)-\left(x_{41}^{2}-x_{31}^{2}\right)}{\left(x_{46}^{2}-x_{36}^{2}\right) t+\left(x_{41}^{2}-x_{31}^{2}\right) \bar{t}} \ln \frac{x_{41}^{2} \bar{t}+x_{46}^{2} t}{x_{31}^{2} \bar{t}+x_{36}^{2} t}+O\left(\epsilon^{0}\right) .
$$

The integral on the right-hand side of this relation can be expressed in terms of polylogarithms of degree 3. Symmetrizing $I_{\mathrm{aux}}^{(\mathrm{b})}$ with respect to cyclic permutations of indices, $i \rightarrow i+1$, and flips, $i \rightarrow 7-i$, we arrive at (46).

Let us now compare the expressions for the two diagrams, Eqs. (A.5) and (A.9). We observe that both $I_{\mathrm{aux}}^{(\mathrm{b})}$ and $I_{\mathrm{NA}}^{c}$ contain a single pole $1 / \epsilon$, but it disappears in the combination $I_{\mathrm{NA}}^{c}-2 I_{\mathrm{aux}}^{(\mathrm{b})}$. This suggests writing $I_{\mathrm{NA}}^{c}$ as

$$
I_{\mathrm{NA}}^{c}=2 I_{\mathrm{aux}}^{(\mathrm{b})}+I_{\text {fin }}^{c}+O(\epsilon)
$$

with $I_{\text {fin }}^{c}$ finite as $\epsilon \rightarrow 0$. Combining (A.5) and (A.9) we find

$$
\begin{aligned}
I_{\text {fin }}^{c} & =-\frac{1}{8}\left(\frac{g^{2}}{4 \pi^{2}}\right)^{2} C_{F} N\left(\frac{x_{46}^{2}}{x_{26}^{2}}-\frac{x_{36}^{2}}{x_{26}^{2}}-\frac{x_{14}^{2}}{x_{26}^{2}}+\frac{x_{13}^{2}}{x_{26}^{2}}\right) \\
& \times \int_{0}^{1} \frac{d t \ln \left(X_{1}(t) / X_{2}(t)\right)}{X_{1}(t)-X_{2}(t)}\left[\frac{1}{2} \ln t \ln \left(X_{1}(t) X_{2}(t) / t^{2}\right)+\int_{t}^{1} \frac{d y}{y} \ln (1-y)\right],
\end{aligned}
$$

where

$$
X_{1}(t)=\frac{x_{14}^{2}}{x_{26}^{2}}(1-t)+\frac{x_{46}^{2}}{x_{26}^{2}} t, \quad X_{2}(t)=\frac{x_{13}^{2}}{x_{26}^{2}}(1-t)+\frac{x_{36}^{2}}{x_{26}^{2}} t .
$$

Taken together, the relations (A.10), (A.11) and (A.9) define the contribution of the diagram shown in Fig. 2(c) to $\ln W\left(C_{6}\right)$. Symmetrization of $I_{\text {fin }}^{c}$ with respect to cyclic permutations of indices and flips yields the expression for $\widehat{A}_{0}^{(\mathrm{c})}$ defined in (50). 
We observe that both the divergent and finite parts of $I_{\mathrm{NA}}^{c}$, Eq. (A.10), are complicated dimensionless functions of four ratios $x_{46}^{2} / x_{26}^{2}, x_{36}^{2} / x_{26}^{2}, x_{14}^{2} / x_{26}^{2}$ and $x_{13}^{2} / x_{26}^{2}$. As such, they are not invariant under conformal transformations of the coordinates $x_{i}^{\mu}$ (with $i=1, \ldots, 6$ ). Moreover, close examination of $I_{\text {aux }}^{(\mathrm{b})}$ and $I_{\text {fin }}^{c}$ shows that the integrals entering the relations (A.9) and (A.11) have transcendentality 3 and 4, respectively. Assigning one unit of the transcendentality to $1 / \epsilon$ we find that $I_{\mathrm{NA}}^{c}$ has degree of transcendentality 4 .

We will now summarize the remaining non-trivial contributions to the finite part of $\ln W_{6}$. Each one must be included in all of its inequivalent orientations. Diagrams (a), (r) and (s) are identical to those appearing in the calculation of the rectangular Wilson loop and they contribute only a constant contribution to the finite part. Their explicit expressions can be found in [19, 20]. The combinations $(\mathrm{m})+(\mathrm{t})$ and $(\mathrm{n})+(\mathrm{u})$ also do not contribute to the finite part, as described in [21]. For the remaining diagrams, some of the contributions (diagrams shown in Fig.2 (h),(k),(l)) factorize into products of finite one-loop integrals given by

$$
\begin{aligned}
J_{j k} & =\int_{0}^{1} \frac{d t_{j} d t_{k}\left(x_{j, j+1} x_{k, k+1}\right)}{\left(x_{j k}-x_{j, j+1} t_{j}+x_{k, k+1} t_{k}\right)^{2}} \\
& =-\frac{1}{2} \int_{0}^{1} d t \frac{\left(x_{j+1, k+1}^{2}-x_{j, k+1}^{2}\right)-\left(x_{j+1, k}^{2}-x_{j k}^{2}\right)}{\left(x_{j+1, k+1}^{2}-x_{j, k+1}^{2}\right) t+\left(x_{j+1, k}^{2}-x_{j k}^{2}\right) \bar{t}} \ln \frac{x_{j+1, k+1}^{2} t+x_{j+1, k}^{2} \bar{t}}{x_{j, k+1}^{2} t+x_{j, k}^{2} \bar{t}} .
\end{aligned}
$$

For the remaining expressions we will often make use of the following shorthand notation for certain ratios of the variables $x_{i j}^{2}$,

$$
\alpha=\frac{x_{26}^{2}}{x_{13}^{2}}, \quad \beta=\frac{x_{36}^{2}}{x_{13}^{2}}, \quad \gamma=\frac{x_{14}^{2}}{x_{13}^{2}}, \quad \delta=\frac{x_{46}^{2}}{x_{13}^{2}}, \quad \eta=\frac{x_{15}^{2}}{x_{13}^{2}} .
$$

Sometimes it is also convenient to use the notation (recall that $p_{i}=x_{i}-x_{i+1}$ )

$$
s_{i j}=2 p_{i} \cdot p_{j}=2 x_{i, i+1} \cdot x_{j, j+1}=x_{i, j+1}^{2}+x_{i+1, j}^{2}-x_{i j}^{2}-x_{i+1, j+1}^{2} .
$$

Further, we will often use the notation $\bar{y} \equiv 1-y$ for some variable $y$. We will never use the bar to refer to complex conjugation. Using this notation, the remaining non-trivial contributions are:

\section{Diagram (b)}

$$
I_{\text {fin }}^{b}=-\frac{1}{8}\left(\frac{g^{2}}{4 \pi^{2}}\right)^{2} C_{F} N \int_{0}^{1} \frac{d t}{t+s_{12} / s_{26}}\left[\frac{1}{2} \ln ^{2} X(t) \ln t+\ln X(t) \int_{t}^{1} \frac{d y}{y} \ln \bar{y}\right],
$$

where $X(t)=1 /(\alpha t)+1+s_{26} / s_{16}$.

\section{Diagram (d)}

$$
I_{\text {fin }}^{d}=-\frac{1}{8}\left(\frac{g^{2}}{4 \pi^{2}}\right)^{2} C_{F} N \int_{0}^{1} \frac{d t \ln \left(X_{1} / X_{2}\right)}{X_{1}-X_{2}}\left[\frac{1}{2} \ln t \ln \left(\frac{X_{1} X_{2} s_{46}^{2}}{t^{2} s_{16}^{2}}\right)+\int_{t}^{1} \frac{d y}{y} \ln \bar{y}\right] \text {, }
$$

where $X_{1}=\left(x_{51}^{2} \bar{t}\right) /\left(x_{41}^{2}-x_{46}^{2}-x_{51}^{2}\right)$ and $X_{2}=\left(x_{41}^{2} \bar{t}+x_{46}^{2} t\right) /\left(x_{41}^{2}-x_{46}^{2}-x_{51}^{2}\right)$. 


\section{Diagram (e)}

$$
I_{\mathrm{fin}}^{e}=\left(\frac{g^{2}}{4 \pi^{2}}\right)^{2} C_{F}\left(-\frac{1}{2} N\right)\left[-\frac{\zeta_{2}}{8} \ln ^{2}\left(\frac{x_{15}^{2}}{x_{26}^{2}}\right)+\frac{19}{1440} \pi^{4}\right] .
$$

\section{Diagram (f)}

$$
\begin{aligned}
I^{f}=\frac{g^{4}}{\left(4 \pi^{2}\right)^{2}} C_{F}\left(-\frac{1}{2} N\right) \frac{1}{4} & \int_{0}^{\mu} d t \int_{0}^{\frac{\lambda}{\mu} t} d v \frac{1}{[\lambda+1-v][\delta-\lambda-\alpha-t-1]} \\
& \times \ln \left[\frac{\lambda(\lambda+1-v)+\alpha(\lambda-v)}{\alpha(\lambda-v)}\right] \ln \left[\frac{\mu(\delta-t)-t(\alpha+\lambda)}{\mu(\lambda+\alpha+1)-t(\alpha+\lambda)}\right],
\end{aligned}
$$

where $\lambda=\beta-\alpha-1$ and $\mu=1+\delta-\gamma-\beta$.

\section{Diagram (g)}

$$
\begin{aligned}
I^{g}=\frac{g^{4}}{\left(8 \pi^{2}\right)^{2}} C_{F}\left(-\frac{1}{2} N\right) & \int_{0}^{\rho} d t \int_{0}^{\frac{\lambda}{\rho} t} d v \frac{1}{[v-\lambda-1][\delta+t]} \\
& \times \ln \left[\frac{\lambda(\lambda+1-v)+\alpha(\lambda-v)}{\alpha(\lambda-v)}\right] \ln \left[\frac{t \eta}{\rho \delta+(\rho+\eta) t}\right] .
\end{aligned}
$$

In the expression for $I^{g}$ the following notation has been used: $\lambda=\beta-\alpha-1$ and $\rho=\gamma-\eta-\delta$.

\section{Diagram (h)}

$$
I^{h}=\frac{g^{4}}{\left(4 \pi^{2}\right)^{2}} C_{F}\left(-\frac{1}{2} N\right) J_{14} J_{26},
$$

where $J_{j k}$ is given by (A.13).

\section{Diagram (i)}

$$
\begin{aligned}
I^{i}=\frac{g^{4}}{\left(8 \pi^{2}\right)^{2}} C_{F}\left(-\frac{1}{2} N\right) & \int_{0}^{\lambda} d v \int_{0}^{\lambda} d t \frac{1}{v+\bar{\lambda}} \frac{1}{t-\alpha} \\
& \times \ln \left[\frac{t(v+\bar{\lambda})+\alpha(\lambda-v)}{\alpha(\lambda-v)}\right] \ln \left[\frac{t}{v(t-\alpha)+t \bar{\lambda}+\lambda \alpha}\right] .
\end{aligned}
$$

In the expression for $I^{i}$ the following notation has been used: $\lambda=1+\alpha-\beta$ and $\bar{\lambda}=1-\lambda$.

\section{Diagram (j)}

$$
\begin{aligned}
I^{j}= & \frac{g^{4}}{\left(8 \pi^{2}\right)^{2}} C_{F}\left(-\frac{1}{2} N\right) \int_{0}^{\mu} d v \int_{0}^{\mu} d t \frac{1}{[\delta-\beta-v][1-\beta-t]} \\
& \times \ln \left[\frac{t(\delta-\beta-v)+\beta \mu+v(1-\beta)}{\beta \mu+v(1-\beta)}\right] \ln \left[\frac{\mu(1-t)-t(\beta-\delta)}{v(1-\beta-t)+\beta \mu-t(\beta-\delta)}\right] .
\end{aligned}
$$

In the expression for $I^{j}$ the following notation has been used: $\mu=1-\beta-\gamma+\delta$. 


\section{Diagram (k)}

$$
I^{k}=\frac{g^{4}}{\left(4 \pi^{2}\right)^{2}} C_{F}\left(-\frac{1}{2} N\right) J_{14} J_{36}
$$

\section{Diagram (l)}

$$
I^{l}=\frac{g^{4}}{\left(4 \pi^{2}\right)^{2}} C_{F}\left(-\frac{1}{2} N\right) J_{15} J_{26}
$$

\section{Diagram (o)}

There are several contributions to the finite part coming from this diagram. We have

$$
I_{\text {fin }}^{o}=I_{\text {fin }, 1}^{o}+I_{\text {fin }, 2}^{o}+I_{\text {fin }, 3}^{o} .
$$

The first is

$$
I_{\mathrm{fin}, 1}^{o}=\left(\frac{g^{2}}{4 \pi^{2}}\right)^{2} \frac{C_{F} N_{c}}{16} \int_{0}^{1} \frac{d x}{x \bar{x}} \int_{0}^{x} d t_{1} \int_{0}^{\bar{x}} d t_{2} \int_{0}^{1} d v\left[\left(\left(\bar{a} t_{1}+a \bar{t}_{2}\right) v+b t_{1} t_{2} \bar{v}\right]^{-1}+(a \longleftrightarrow b)\right] .
$$

The second part is

$$
\begin{gathered}
I_{\text {fin }, 2}^{o}=\left(\frac{g^{2}}{4 \pi^{2}}\right)^{2} \frac{C_{F} N}{16}\left(K_{1}+K_{2}+K_{3}\right), \\
K_{1}=-2(1-a-b) \int_{0}^{1} d t_{1} d t_{3} d x d v \frac{v}{\bar{v}} \frac{a t_{3}+b t_{1}}{x t_{1} b-\bar{x} t_{3} a}\left[\frac{\bar{x}}{\bar{v} b t_{1}+v \bar{x} C}-\frac{x}{\bar{v} a t_{3}+v x C}\right] \\
K_{2}=(1-a-b) \int_{0}^{1} d t_{1} d t_{3} d x d v \frac{1}{x B-\bar{x} A}\left[\frac{B}{B \bar{v}+v \bar{x} C}-\frac{A}{A \bar{v}+v x C}\right] \\
K_{3}=(1-a-b) \int_{0}^{1} d t_{1} d t_{3} d x d v\left\{\frac{1}{x}\left[\frac{\bar{x} A}{x B-\bar{x} A} \frac{1}{B \bar{v}+v \bar{x} C}+\frac{1}{B \bar{v}+v C}\right]\right. \\
\left.+\frac{1}{\bar{x}}\left[-\frac{x B}{x B-\bar{x} A} \frac{1}{A \bar{v}+v x C}+\frac{1}{A \bar{v}+v C}\right]\right\}
\end{gathered}
$$

Here we use the notation,

$$
a=1 / \beta, \quad b=\alpha / \beta, \quad A=a t_{3}, \quad B=b t_{1}, \quad C=a t_{3} \bar{t}_{1}+b t_{1} \bar{t}_{3}+t_{1} t_{3} .
$$

Finally the third part is

$$
I_{\mathrm{fin}, 3}^{o}=\frac{1}{32}\left(\frac{g^{2}}{4 \pi^{2}}\right)^{2} C_{F} N \int_{0}^{1} d t_{3} \frac{s_{26}}{s_{16}+s_{26} t_{3}} \ln \frac{s_{16}+\left(s_{12}+s_{26}\right) t_{3}}{s_{12} t_{3}} \ln ^{2} t_{3}+\left(s_{12} \leftrightarrows s_{16}\right) .
$$




\section{Diagram (p)}

There are several contributions to the finite part coming from this diagram. We have

$$
I_{\mathrm{fin}}^{p}=I_{\mathrm{fin}, 1}^{p}+I_{\mathrm{fin}, 2}^{p}+I_{\mathrm{fin}, 3}^{p} .
$$

The first part is

$$
I_{\mathrm{fin}, 1}^{p}=-\frac{1}{8}\left(\frac{g^{2}}{4 \pi^{2}}\right)^{2} C_{F} N \int_{0}^{1} d v_{2} d v_{3} \int_{0}^{1} d x \int_{0}^{\infty} \frac{d y\left(p_{3} \cdot p_{6}\right)}{(y+1)\left(y x \bar{x} z_{12}^{2}+\bar{x} z_{23}^{2}+x z_{31}^{2}\right)},
$$

with

$$
z_{12}=p_{2}+p_{3} v_{2}, \quad z_{23}=x_{31}-p_{3} v_{2}-p_{6} \bar{v}_{3}, \quad z_{31}=p_{1}+p_{6} \bar{v}_{3} .
$$

Similarly the second is

$$
I_{\mathrm{fin}, 2}^{p}=-\frac{1}{8}\left(\frac{g^{2}}{4 \pi^{2}}\right)^{2} C_{F} N \int_{0}^{1} d v_{1} d v_{3} \int_{0}^{1} d x \int_{0}^{\infty} \frac{d y\left(p_{2} \cdot p_{6}\right)}{(y+1)\left(y x \bar{x} z_{12}^{2}+\bar{x} z_{23}^{2}+x z_{31}^{2}\right)},
$$

with

$$
z_{12}=p_{2} \bar{v}_{1}+p_{3}, \quad z_{23}=x_{41}-p_{6} \bar{v}_{3}, \quad z_{31}=p_{1}+p_{2} v_{1}+p_{6} \bar{v}_{3} .
$$

The final contribution is

$$
I_{\mathrm{fin}, 3}^{p}=\frac{1}{8}\left(\frac{g^{2}}{4 \pi^{2}}\right)^{2} C_{F} N \int_{0}^{1} d v_{1} d v_{2} d v_{3} \int_{0}^{1} d x \int_{0}^{\infty} \frac{d y}{1+y} \frac{x V_{31}+\bar{x} V_{23}}{\left[y x \bar{x} z_{12}^{2}+\bar{x} z_{23}^{2}+x z_{31}^{2}\right]^{2}},
$$

where

$$
z_{12}=p_{2} \bar{v}_{1}+p_{3} v_{2}, \quad z_{23}=x_{31}-p_{3} v_{2}-p_{6} \bar{v}_{3}, \quad z_{31}=p_{1}+p_{2} v_{1}+p_{6} \bar{v}_{3}=-x_{31}-p_{2} \bar{v}_{1}+p_{6} \bar{v}_{3}
$$

and

$$
\begin{aligned}
& V_{23}=-2\left(p_{2} \cdot p_{3}\right)\left(p_{6} \cdot x_{31}\right)+4\left(p_{3} \cdot p_{6}\right)\left(p_{2} \cdot x_{31}\right)-2\left(p_{2} \cdot p_{3}\right)\left(p_{3} \cdot p_{6}\right) v_{2}-4\left(p_{3} \cdot p_{6}\right)\left(p_{2} \cdot p_{6}\right) \bar{v}_{3} \\
& V_{31}=-2\left(p_{2} \cdot p_{3}\right)\left(p_{1} \cdot p_{6}\right)+4\left(p_{1} \cdot p_{3}\right)\left(p_{2} \cdot p_{6}\right)+2\left(p_{2} \cdot p_{3}\right)\left(p_{2} \cdot p_{6}\right) v_{1}+4\left(p_{2} \cdot p_{6}\right)\left(p_{3} \cdot p_{6}\right) \bar{v}_{3}
\end{aligned}
$$

\section{Diagram (q)}

$$
\begin{aligned}
I^{q}=\frac{g^{4} C_{F} N}{128 \pi^{4}} & \int_{0}^{1} d t_{1} d t_{3} d t_{5} \delta\left(t_{1}+t_{3}+t_{5}-1\right) \int_{0}^{1} \prod_{i} d v_{i}\left[t_{1} t_{3} z_{13}^{2}+t_{3} t_{5} z_{35}^{2}+t_{5} t_{1} z_{51}^{2}\right]^{-2} \\
& \times \Gamma_{3}\left(\partial_{z_{1}}, \partial_{z_{3}}, \partial_{z_{5}}\right)\left[t_{1} t_{3} z_{13}^{2}+t_{3} t_{5} z_{35}^{2}+t_{5} t_{1} z_{51}^{2}\right]
\end{aligned}
$$

Here $\Gamma_{3}$ is the differential operator,

$\Gamma_{3}\left(\partial_{z_{1}}, \partial_{z_{3}}, \partial_{z_{5}}\right)=-\left(p_{1} \cdot p_{3}\right)\left(p_{5} \cdot\left(\partial_{z_{3}}-\partial_{z_{1}}\right)\right)-\left(p_{3} \cdot p_{5}\right)\left(p_{1} \cdot\left(\partial_{z_{5}}-\partial_{z_{3}}\right)\right)-\left(p_{5} \cdot p_{1}\right)\left(p_{3} \cdot\left(\partial_{z_{1}}-\partial_{z_{5}}\right)\right)$, and the $z_{j k}^{2}$ variables are defined by

$$
z_{13}^{2}=\left(p_{1} \bar{v}_{1}+p_{2}+p_{3} v_{3}\right)^{2}, \quad z_{35}^{2}=\left(p_{3} \bar{v}_{3}+p_{4}+p_{5} v_{5}\right)^{2}, \quad z_{51}^{2}=\left(p_{5} \bar{v}_{5}+p_{6}+p_{1} v_{1}\right)^{2} .
$$




\section{References}

[1] L. F. Alday and J. Maldacena, "Gluon scattering amplitudes at strong coupling," JHEP 0706 (2007) 064 [arXiv:0705.0303 [hep-th]].

[2] Z. Bern, L. J. Dixon, D. C. Dunbar and D. A. Kosower "One loop n point gauge theory amplitudes, unitarity and collinear limits," Nucl. Phys. B 425, 217 (1994) arXiv:hep-ph/9403226].

[3] Z. Bern, J. S. Rozowsky and B. Yan, "Two-loop four-gluon amplitudes in N= 4 super-YangMills," Phys. Lett. B 401 (1997) 273 arXiv:hep-ph/9702424.

[4] Z. Bern, L. J. Dixon, D. C. Dunbar and D. A. Kosower, "Fusing gauge theory tree amplitudes into loop amplitudes," Nucl. Phys. B 435, 59 (1995) arXiv:hep-ph/9409265];

Z. Bern, L. J. Dixon and D. A. Kosower, "Two-loop g $\rightarrow$ g g splitting amplitudes in QCD," JHEP 0408, 012 (2004) arXiv:hep-ph/0404293;

R. Britto, F. Cachazo and B. Feng, "Computing one-loop amplitudes from the holomorphic anomaly of unitarity cuts," Phys. Rev. D 71, 025012 (2005) arXiv:hep-th/0410179;

Z. Bern, V. Del Duca, L. J. Dixon and D. A. Kosower, "All non-maximally-helicity-violating one-loop seven-gluon amplitudes in $\mathrm{N}=4$ super-Yang-Mills theory," Phys. Rev. D 71, 045006 (2005) arXiv:hep-th/0410224;

R. Britto, F. Cachazo and B. Feng, "Generalized unitarity and one-loop amplitudes in $\mathrm{N}=$ 4 super-Yang-Mills," Nucl. Phys. B 725, 275 (2005) arXiv:hep-th/0412103;

Z. Bern, L. J. Dixon and D. A. Kosower, "All next-to-maximally helicity-violating oneloop gluon amplitudes in $\mathrm{N}=4$ super-Yang-Mills theory," Phys. Rev. D 72, 045014 (2005) arXiv:hep-th/0412210.

[5] Z. Bern, L. J. Dixon and V. A. Smirnov, "Iteration of planar amplitudes in maximally supersymmetric Yang-Mills theory at three loops and beyond," Phys. Rev. D 72 (2005) 085001 [arXiv:hep-th/0505205].

[6] C. Anastasiou, Z. Bern, L. J. Dixon and D. A. Kosower, "Planar amplitudes in maximally supersymmetric Yang-Mills theory," Phys. Rev. Lett. 91, 251602 (2003) arXiv:hep-th/0309040.

[7] R. Akhoury, "Mass divergences of wide angle scattering amplitudes," Phys. Rev. D 19 (1979) 1250 ;

A. H. Mueller, "On the asymptotic behavior of the Sudakov form-factor," Phys. Rev. D 20 (1979) 2037;

J. C. Collins, "Algorithm to compute corrections to the Sudakov form-factor," Phys. Rev.

D 22 (1980) 1478;

A. Sen, "Asymptotic behavior of the sudakov form-factor in QCD," Phys. Rev. D 24 (1981) 3281 ;

G. Sterman, "Summation of large corrections to short distance hadronic cross-sections," Nucl. Phys. B 281 (1987) 310;

J. Botts and G. Sterman, "Hard elastic scattering in QCD: leading behavior," Nucl. Phys.

B 325 (1989) 62;

S. Catani and L. Trentadue, "Resummation of the QCD perturbative series for hard processes," Nucl. Phys. B 327 (1989) 323; 
G. P. Korchemsky, "Sudakov form-factor in QCD," Phys. Lett. B 220 (1989) 629; "Double logarithmic asymptotics in QCD," Phys. Lett. B 217 (1989) 330;

L. Magnea and G. Sterman, "Analytic continuation of the Sudakov form-factor in QCD," Phys. Rev. D 42 (1990) 4222;

G. P. Korchemsky and G. Marchesini, "Resummation of large infrared corrections using Wilson loops," Phys. Lett. B 313 (1993) 433;

S. Catani, "The singular behaviour of QCD amplitudes at two-loop order," Phys. Lett. B 427 (1998) 161 arXiv:hep-ph/9802439;

G. Sterman and M. E. Tejeda-Yeomans, "Multi-loop amplitudes and resummation," Phys. Lett. B 552 (2003) 48 [arXiv:hep-ph/0210130].

[8] G. P. Korchemsky and A. V. Radyushkin, "Loop Space Formalism And Renormalization Group For The Infrared Asymptotics Of QCD," Phys. Lett. B 171 (1986) 459;

S. V. Ivanov, G. P. Korchemsky and A. V. Radyushkin, "Infrared Asymptotics Of Perturbative QCD: Contour Gauges," Yad. Fiz. 44 (1986) 230 [Sov. J. Nucl. Phys. 44 (1986) 145].

[9] G. P. Korchemsky and G. Marchesini, "Structure function for large x and renormalization of Wilson loop," Nucl. Phys. B 406 (1993) 225 arXiv:hep-ph/9210281.

[10] I. A. Korchemskaya and G. P. Korchemsky, "Evolution equation for gluon Regge trajectory," Phys. Lett. B 387 (1996) 346 arXiv:hep-ph/9607229].

[11] A. V. Belitsky, A. S. Gorsky and G. P. Korchemsky, "Gauge / string duality for QCD conformal operators," Nucl. Phys. B 667 (2003) 3 arXiv:hep-th/0304028.

[12] F. Cachazo, M. Spradlin and A. Volovich, "Iterative structure within the five-particle twoloop amplitude," Phys. Rev. D 74 (2006) 045020 arXiv:hep-th/0602228;

Z. Bern, M. Czakon, D. A. Kosower, R. Roiban and V. A. Smirnov, "Two-loop iteration of five-point N = 4 super-Yang-Mills amplitudes," Phys. Rev. Lett. 97 (2006) 181601 arXiv:hep-th/0604074.

[13] Z. Bern, L. J. Dixon, D. A. Kosower, R. Roiban, M. Spradlin, C. Vergu and A. Volovich, "The two-loop six-gluon MHV amplitude in maximally supersymmetric Yang-Mills theory," arXiv:0803.1465 [hep-th].

[14] L. F. Alday and J. Maldacena, "Comments on gluon scattering amplitudes via AdS/CFT," JHEP 0711 (2007) 068 [arXiv:0710.1060 [hep-th]].

[15] J. M. Maldacena, "Wilson loops in large N field theories," Phys. Rev. Lett. 80 (1998) 4859 arXiv:hep-th/9803002;

S. J. Rey and J. T. Yee, "Macroscopic strings as heavy quarks in large N gauge theory and anti-de Sitter supergravity," Eur. Phys. J. C 22 (2001) 379 [arXiv:hep-th/9803001].

[16] M. Kruczenski, "A note on twist two operators in N = 4 SYM and Wilson loops in Minkowski signature," JHEP 0212 (2002) 024 arXiv:hep-th/0210115;

$\mathrm{Yu}$. Makeenko, "Light-cone Wilson loops and the string/gauge correspondence," JHEP 0301 (2003) 007 arXiv:hep-th/0210256. 
[17] J. M. Drummond, G. P. Korchemsky and E. Sokatchev, "Conformal properties of fourgluon planar amplitudes and Wilson loops," Nucl. Phys. B 795 (2008) 385 arXiv:0707.0243 [hep-th]].

[18] A. Brandhuber, P. Heslop and G. Travaglini, "MHV Amplitudes in N=4 Super Yang-Mills and Wilson Loops," Nucl. Phys. B 794 (2008) 231 [arXiv:0707.1153 [hep-th]].

[19] I. A. Korchemskaya and G. P. Korchemsky, "On light-like Wilson loops," Phys. Lett. B 287 (1992) 169;

A. Bassetto, I. A. Korchemskaya, G. P. Korchemsky and G. Nardelli, "Gauge invariance and anomalous dimensions of a light cone Wilson loop in lightlike axial gauge," Nucl. Phys. B 408 (1993) 62 arXiv:hep-ph/9303314.

[20] J. M. Drummond, J. Henn, G. P. Korchemsky and E. Sokatchev, "On planar gluon amplitudes/Wilson loops duality," Nucl. Phys. B 795 (2008) 52 [arXiv:0709.2368 [hep-th]].

[21] J. M. Drummond, J. Henn, G. P. Korchemsky and E. Sokatchev, "Conformal Ward identities for Wilson loops and a test of the duality with gluon amplitudes," arXiv:0712.1223 [hep-th].

[22] L. F. Alday and J. Maldacena, private communication.

[23] Z. Komargodski, "On collinear factorization of Wilson loops and MHV amplitudes in N=4 SYM," arXiv:0801.3274 [hep-th].

[24] J. M. Drummond, J. Henn, G. P. Korchemsky and E. Sokatchev, "The hexagon Wilson loop and the BDS ansatz for the six-gluon amplitude," arXiv:0712.4138 [hep-th].

[25] J. McGreevy and A. Sever, "Quark scattering amplitudes at strong coupling," JHEP 0802 (2008) 015 [arXiv:0710.0393 [hep-th]].

[26] Z. Komargodski and S. S. Razamat, "Planar quark scattering at strong coupling and universality," JHEP 0801 (2008) 044 [arXiv:0707.4367 [hep-th]].

[27] Y. Oz, S. Theisen and S. Yankielowicz, "Gluon Scattering in Deformed N=4 SYM," arXiv:0712.3491 [hep-th].

[28] A. V. Kotikov and L. N. Lipatov, "NLO corrections to the BFKL equation in QCD and in supersymmetric gauge theories," Nucl. Phys. B 582 (2000) 19 arXiv:hep-ph/0004008.

[29] S. G. Naculich and H. J. Schnitzer, "Regge behavior of gluon scattering amplitudes in N=4 SYM theory," Nucl. Phys. B 794 (2008) 189 [arXiv:0708.3069 [hep-th]].

[30] R. C. Brower, H. Nastase, H. J. Schnitzer and C. I. Tan, "Implications of multi-Regge limits for the Bern-Dixon-Smirnov conjecture," arXiv:0801.3891 [hep-th].

[31] J. Bartels, L. N. Lipatov and A. S. Vera, "BFKL Pomeron, Reggeized gluons and BernDixon-Smirnov amplitudes," arXiv:0802.2065 [hep-th].

[32] V. Del Duca and E. W. N. Glover, "Testing high-energy factorization beyond the next-toleading-logarithmic accuracy," arXiv:0802.4445 [hep-th]. 
[33] M. L. Mangano and S. J. Parke, "Multiparton amplitudes in gauge theories," Phys. Rept. 200 (1991) 301 arXiv:hep-th/0509223.

[34] M. T. Grisaru, H. N. Pendleton and P. van Nieuwenhuizen, "Supergravity And The S Matrix," Phys. Rev. D 15 (1977) 996;

M. T. Grisaru and H. N. Pendleton, "Some Properties Of Scattering Amplitudes In Supersymmetric Theories," Nucl. Phys. B 124 (1977) 81;

S. J. Parke and T. R. Taylor, "Perturbative QCD Utilizing Extended Supersymmetry," Phys. Lett. B 157 (1985) 81 [Erratum-ibid. 174B (1986) 465].

[35] Z. Bern, L. J. Dixon, D. C. Dunbar and D. A. Kosower, "One-loop self-dual and N = 4 superYang-Mills," Phys. Lett. B 394, 105 (1997) arXiv:hep-th/9611127.

[36] Z. Bern and D. A. Kosower, "The Computation of loop amplitudes in gauge theories," Nucl. Phys. B 379, 451 (1992);

Z. Bern, A. De Freitas, L. J. Dixon and H. L. Wong, "Supersymmetric regularization, two-loop QCD amplitudes and coupling shifts," Phys. Rev. D 66, 085002 (2002) arXiv:hep-ph/0202271.

[37] A. M. Polyakov, "Gauge Fields As Rings Of Glue," Nucl. Phys. B 164 (1980) 171.

[38] G. P. Korchemsky and A. V. Radyushkin, "Renormalization of the Wilson Loops Beyond the Leading Order," Nucl. Phys. B 283 (1987) 342.

[39] Z. Bern, M. Czakon, L. J. Dixon, D. A. Kosower and V. A. Smirnov, "The Four-Loop Planar Amplitude and Cusp Anomalous Dimension in Maximally Supersymmetric YangMills Theory," Phys. Rev. D 75 (2007) 085010 arXiv:hep-th/0610248;

F. Cachazo, M. Spradlin and A. Volovich, "Four-Loop Cusp Anomalous Dimension From Obstructions," Phys. Rev. D 75, (2007) 105011 [arXiv:hep-th/0612309].

[40] N. Beisert, B. Eden and M. Staudacher, "Transcendentality and crossing," J. Stat. Mech. 0701 (2007) P021 arXiv:hep-th/0610251.

[41] M. K. Benna, S. Benvenuti, I. R. Klebanov and A. Scardicchio, "A test of the AdS/CFT correspondence using high-spin operators," Phys. Rev. Lett. 98 (2007) 131603 arXiv:hep-th/0611135;

L. F. Alday, G. Arutyunov, M. K. Benna, B. Eden and I. R. Klebanov, JHEP 0704 (2007) 082 arXiv:hep-th/0702028.

[42] B. Basso, G. P. Korchemsky and J. Kotanski, "Cusp anomalous dimension in maximally supersymmetric Yang-Mills theory at strong coupling," arXiv:0708.3933 [hep-th].

[43] I. Kostov, D. Serban and D. Volin, "Functional BES equation," arXiv:0801.2542 [hep-th].

[44] S. S. Gubser, I. R. Klebanov and A. M. Polyakov, "A semi-classical limit of the gauge/string correspondence," Nucl. Phys. B 636 (2002) 99 arXiv:hep-th/0204051.

[45] S. Frolov and A. A. Tseytlin, "Semiclassical quantization of rotating superstring in AdS(5) x S(5)," JHEP 0206 (2002) 007 arXiv:hep-th/0204226]. 
[46] R. Roiban and A. A. Tseytlin, "Strong-coupling expansion of cusp anomaly from quantum superstring," JHEP 0711 (2007) 016 [arXiv:0709.0681 [hep-th]].

[47] F. Cachazo, M. Spradlin and A. Volovich, "Four-Loop Collinear Anomalous Dimension in N = 4 Yang-Mills Theory," Phys. Rev. D 76 (2007) 106004 [arXiv:0707.1903 [hep-th]].

[48] V. S. Dotsenko and S. N. Vergeles, "Renormalizability Of Phase Factors In The Nonabelian Gauge Theory," Nucl. Phys. B 169 (1980) 527;

R. A. Brandt, F. Neri and M. a. Sato, "Renormalization Of Loop Functions For All Loops," Phys. Rev. D 24 (1981) 879;

H. Dorn, "Renormalization of path ordered phase factors and related hadron operators in gauge field theories," Fortsch. Phys. 34 (1986) 11.

[49] L. J. Dixon, L. Magnea and G. Sterman, "Universal structure of subleading infrared poles in gauge theory amplitudes," JHEP 0808 (2008) 022 [arXiv:0805.3515 [hep-ph]].

[50] Z. Bern, J. J. M. Carrasco, H. Johansson and D. A. Kosower, "Maximally supersymmetric planar Yang-Mills amplitudes at five loops," Phys. Rev. D 76 (2007) 125020 arXiv:0705.1864 [hep-th]].

[51] D. J. Broadhurst, "Summation of an infinite series of ladder diagrams," Phys. Lett. B 307 (1993) 132;

J. M. Drummond, J. Henn, V. A. Smirnov and E. Sokatchev, "Magic identities for conformal four-point integrals," JHEP 0701 (2007) 064 [arXiv:hep-th/0607160].

[52] F. Cachazo and D. Skinner, "On the structure of scattering amplitudes in N=4 super YangMills and $\mathrm{N}=8$ supergravity," arXiv:0801.4574 [hep-th].

[53] G. Sterman, in AIP Conference Proceedings Tallahassee, Perturbative Quantum Chromodynamics, eds. D. W. Duke, J. F. Owens, New York, 1981, p. 22;

J. G. M. Gatheral, "Exponentiation Of Eikonal Cross-Sections In Nonabelian Gauge Theories," Phys. Lett. B 133 (1983) 90;

J. Frenkel and J. C. Taylor, "Nonabelian Eikonal Exponentiation," Nucl. Phys. B 246 (1984) 231.

[54] A. V. Kotikov, L. N. Lipatov, A. I. Onishchenko and V. N. Velizhanin, "Three-loop universal anomalous dimension of the Wilson operators in N = 4 SUSY Yang-Mills model," Phys. Lett. B 595 (2004) 521 [Erratum-ibid. B 632 (2006) 754] arXiv:hep-th/0404092.

[55] E. Witten, "Perturbative gauge theory as a string theory in twistor space," Commun. Math. Phys. 252 (2004) 189 arXiv:hep-th/0312171].

[56] Yu. M. Makeenko and A. A. Migdal, "Exact Equation For The Loop Average In Multicolor QCD," Phys. Lett. B 88 (1979) 135 [Erratum-ibid. B 89 (1980) 437].

[57] N. Drukker, D. J. Gross and H. Ooguri, "Wilson loops and minimal surfaces," Phys. Rev. D 60 (1999) 125006 [arXiv:hep-th/9904191]. 
[58] A. M. Polyakov and V. S. Rychkov, "Gauge fields - strings duality and the loop equation," Nucl. Phys. B 581 (2000) 116 arXiv:hep-th/0002106];

A. M. Polyakov and V. S. Rychkov, "Loop dynamics and AdS/CFT correspondence," Nucl. Phys. B 594 (2001) 272 [arXiv:hep-th/0005173]. 